OPEN ACCESS

Edited by:

Akio Adachi,

Tokushima University, Japan

Reviewed by:

Masato Tsurudome,

Mie University, Japan

Takemasa Sakaguchi,

Hiroshima University, Japan

*Correspondence:

Guo-zhong Zhang

zhanggz@cau.edu.cn

Specialty section: This article was submitted to Virology,

a section of the journal

Frontiers in Microbiology

Received: 26 June 2017 Accepted: 07 August 2017

Published: 23 August 2017

Citation:

Jin J-h, Cheng J-I, He Z-r, Ren Y-c, $Y u X-h$, Song $Y$, Yang $H-m$, Yang $Y-I$, Liu T and Zhang G-Z (2017) Different

Origins of Newcastle

Disease Virus Hemagglutinin-

Neuraminidase Protein Modulate

the Replication Efficiency

and Pathogenicity of the Virus.

Front. Microbiol. 8:1607.

doi: 10.3389/fmicb.2017.01607

\section{Different Origins of Newcastle Disease Virus Hemagglutinin- Neuraminidase Protein Modulate the Replication Efficiency and Pathogenicity of the Virus}

\author{
Ji-hui Jin', Jin-long Cheng ${ }^{1}$, Zi-rong He ${ }^{1}$, Ying-chao Ren², Xiao-hui Yu' ${ }^{1}$, Yang Song', \\ Hui-ming Yang ${ }^{1}$, Yan-ling Yang ${ }^{1}$, Tong Liu' ${ }^{1}$ and Guo-zhong Zhang ${ }^{1,2 *}$ \\ 1 Key Laboratory of Animal Epidemiology of the Ministry of Agriculture, College of Veterinary Medicine, China Agricultural \\ University, Beijing, China, ${ }^{2}$ Diagnostic and Research Center of Livestock and Poultry Epidemic Diseases, China Agricultural \\ University, Beijing, China
}

To investigate the exact effects of different origins of Newcastle disease virus (NDV) hemagglutinin-neuraminidase $(\mathrm{HN})$ protein to the biological characteristics of the virus, we systematically studied the correlation between the HN protein and NDV virulence by exchanging the HN of velogenic or lentogenic NDV strains with the HN from other strains of different virulence. The results revealed that the rSG10 or rLaSota derivatives bearing the HN gene of other viruses exhibited decreased or increased hemadsorption (HAd), neuraminidase and fusion promotion activities. In vitro and in vivo tests further showed that changes in replication level, tissue tropism and virulence of the chimeric viruses were also consistent with these biological activities. These findings demonstrated that the balance among three biological activities caused variation in replication and pathogenicity of the virus, which was closely related to the origin of the HN protein. Our study highlights the importance of the HN glycoprotein in modulating the virulence of NDV and contributes to a more complete understanding of the virulence of NDV.

Keywords: Newcastle disease virus, hemagglutinin-neuraminidase protein, reverse genetics, biological activity, replication, pathogenicity

\section{INTRODUCTION}

Newcastle disease (ND) is a constant threat to the poultry industry worldwide and has caused severe economic losses. The causative pathogen is Newcastle disease virus (NDV), which is a member of the genus Avulavirus in the family Paramyxoviridae (Mayo, 2002). NDV strains are classified into three major pathotypes: lentogenic, mesogenic, and velogenic, based on their pathogenicity in chickens. NDV can be classified into two classes based on genome length and the sequence of the F gene. Class I viruses are less genetically diverse, are generally present in wild waterfowl, and are of low virulence (Dimitrov et al., 2016). Class II can be further divided into nine genotypes (Munir et al., 2012). Among these, genotype VII NDVs are the predominant strains isolated throughout the world in recent years (Miller et al., 2009). Genotype VI viruses, sometimes 
referred to as pigeon paramyxovirus serotype 1, are generally of moderate virulence (Dortmans et al., 2009). Genotype II within class II includes isolates of high and low virulence, and are often used as vaccine strains. Strains of avian paramyxovirus type 2 (APMV-2) is much less virulent and have been associated with asymptomatic to mild respiratory diseases in chickens (Alexander, 1982).

Newcastle disease virus has a non-segmented, single-stranded, negative-sense RNA genome of $15,186,15,192$, or 15,198 nucleotides in length. The genome encodes six structural proteins: nucleoprotein (NP), phosphoprotein (P), matrix protein $(\mathrm{M})$, fusion protein $(\mathrm{F})$, hemagglutinin-neuraminidase $(\mathrm{HN})$ and large polymerase protein (L), transcribed from six genes in the following order: $3^{\prime}$-NP-P-M-F-HN-L-5'. RNA editing of the $\mathrm{P}$ protein produces additional non-structural proteins V and W (Miller and Koch, 2013). NDV infection is initiated by receptor recognition and binding to the host cell surface, followed by fusion, which is accomplished by the interaction of $\mathrm{F}$ and $\mathrm{HN}$ proteins (Connaris et al., 2002).

Though the amino acid sequence at the $\mathrm{F}$ protein cleavage site has been identified as the primary determinant of $\mathrm{NDV}$ virulence, the NDV $\mathrm{HN}$ protein plays an important role in viral invasion and maturation, and is present on the surface of virions and infected cells (Peeters et al., 1999; Panda et al., 2004; Jin et al., 2016). HN is a type II homotetrameric glycoprotein consisting of an N-terminal transmembrane domain and an ectodomain, which has a globular head perched on top of a membrane-anchored stalk domain (Yuan et al., 2012). HN is a multifunctional molecule with three distinct activities: receptor binding, neuraminidase (NA) activity and fusion promotion (Mirza et al., 1994; Melanson and Iorio, 2004). The globular head includes two receptorbinding sites, site I is associated with receptor binding and neuraminidase activity, site II is involved in receptor binding and fusion (Crennell et al., 2000; Iorio et al., 2001; Zaitsev et al., 2004; Jin et al., 2016). In addition, the stalk domain promotes membrane fusion through its interaction with the $\mathrm{F}$ protein (Melanson and Iorio, 2004; Porotto et al., 2012). The HN protein has been shown to contribute greatly to NDV pathogenesis (Huang et al., 2004; Kim et al., 2011; Cornax et al., 2013).

Several studies have investigated the contribution of the $\mathrm{HN}$ gene to NDV virulence and tropism by exchanging genes between strains (Huang et al., 2004; Oldoni et al., 2005; Wakamatsu et al., 2006; Paldurai et al., 2014). Although these studies have increased our understanding of the role of $\mathrm{HN}$ in NDV virulence, some of the results have been controversial and conflicting. The $\mathrm{HN}$ from a lentogenic virus (LaSota) inserted into a virulent backbone (Beaudette C; BC) caused dissemination of virus in a manner similar to wild type virulent virus (BC) (Oldoni et al., 2005). On the contrary, the LaSota $\mathrm{HN}$ within the BC backbone decreased disease severity and pathogenicity indices (Huang et al., 2004; Wakamatsu et al., 2006). Presence of $\mathrm{HN}$ from virulent virus BC inserted into a LaSota backbone caused dissemination of virus in a manner similar to BC (Huang et al., 2004; Oldoni et al., 2005). However, the $\mathrm{BC} \mathrm{HN}$ within the LaSota backbone did not change either severity of disease in chickens or pathogenicity indices (Wakamatsu et al., 2006). Furthermore, the HN genes of a velogenic NDV strain GB Texas (GBT) and the mesogenic BC were exchanged, but the tropism and virulence of the chimeric recombinant viruses were not obviously altered (Paldurai et al., 2014).

In this study, we conducted a systematic study of the contribution of the $\mathrm{HN}$ protein to $\mathrm{NDV}$ virulence and pathogenesis by replacing the complete $\mathrm{HN}$ open reading frame (ORF) of a velogenic genotype VII SG10 strain and a lentogenic genotype II LaSota strain, with $\mathrm{HN}$ proteins of different origins. The complete HN ORF of strains BJ (a mesogenic genotype VI NDV), LaSota, HB (a lentogenic class I NDV) and Yucaipa (a lentogenic APMV-2 strain) were inserted into the rSG10 backbone and the complete HN ORF of rLaSota was replaced with the corresponding HN ORF of strains SG10, BJ, HB and Yucaipa. A total of seven chimeric viruses, plus the two parent strains, were recovered. We evaluated the viral replication in DF-1 cells and 3-week-old specific-pathogen-free (SPF) chickens, virulence in 9-day-old SPF chicken eggs, 1-day-old SPF chicks and 6-week-old SPF chickens, pathogenicity in 3-week-old SPF chickens, and HN biological activities at viral and protein levels in vitro.

\section{MATERIALS AND METHODS}

\section{Animals and Ethics Statement}

Specific-pathogen-free chickens and embryonated eggs were purchased from Merial Vital Laboratory Animal Technology Co., Ltd. (Beijing, China). All birds were kept in isolators and the animal rearing facilities were approved by the Administration Committee of Laboratory Animals under the auspices of the Beijing Association for Science and Technology (approval ID SYXK [Jing] 2013-0013). The experimental protocol, including the possibility of animal death without euthanasia, was specifically considered and approved by the Animal Welfare and Ethical Censor Committee at China Agricultural University (CAU approval number 1605-01).

\section{Cells and Viruses}

Baby hamster kidney (BHK-21) cells stably expressing T7 RNA polymerase (BSR T7), an African green monkey kidney cell line (Vero) and a chicken embryo fibroblast cell line (DF-1) were all grown in Dulbecco's modified eagle medium (DMEM, Gibco, Grand Island, NY, New York) containing 10\% (v/v) fetal bovine serum (FBS, Gibco), and maintained in DMEM containing $2 \%$ FBS at $37^{\circ} \mathrm{C}$ in an incubator (Thermo Forma, Marietta, $\mathrm{OH}$, United States) under 5\% $\mathrm{CO}_{2}$. Recombinant NDV strains rSG10 and rLaSota were generated in our laboratory (Liu M.M. et al., 2015; Yu et al., 2017). The velogenic genotype VII NDV strain SG10, mesogenic genotype VI NDV strain BJ, lentogenic genotype II NDV strain LaSota, lentogenic class I NDV strain $\mathrm{HB}$ and lentogenic APMV-2 strain Yucaipa were propagated in 9-day-old SPF chicken eggs. 


\section{Construction of Full-Length Chimeric SG10/LaSota Antigenomic cDNAs and Generation of Chimeric Viruses}

The HN ORF of genotype VI NDV strain BJ (571 aa), genotype II NDV strain LaSota (572 aa), class I NDV strain HB (616 aa) and APMV-2 strain Yucaipa (580 aa) were individually placed into the full-length antigenomic cDNA of strain rSG10 (571 aa) in place of the corresponding NDV HN ORF via the presence of unique restriction enzyme sites with the Seamless Assembly Cloning Kit (Invitrogen, Carlsbad, CA) (Figure 1A). Firstly, the full-length antigenome of rSG10 was digested with SalI and MluI to generate a single DNA fragment without the HN gene. Then, the HN ORFs of BJ, LaSota, HB and Yucaipa were generated by PCR amplification as DNA fragments each flanked by $5^{\prime}$ and $3^{\prime}$ UTRs of the rSG10 HN gene. To maintain the genome length in multiples of six nucleotides, additional nucleotides were introduced at the $3^{\prime}$ UTR of the HN gene as necessary. The other two DNA fragments with homologous ends, one extending from the SalI site to the HN initiation codon and the other from the HN termination codon to the MluI site, were generated by PCR amplification with compatible primers. Finally, these four DNA fragments with overlapping homologous ends of various lengths were effectively joined together using the cloning kit. The complete HN ORFs of strains SG10, BJ, HB and Yucaipa were inserted into the rLaSota backbone in place of the corresponding rLaSota sequence using the same strategy (Figure 1A). The full-length antigenome of rLaSota was digested with $\mathrm{XbaI}$ and HindIII to generate a single DNA fragment without the $\mathrm{HN}$ gene. All the replaced regions in the full-length cDNAs were sequenced to confirm the presence of the desired genes. The resulting full-length plasmids of rSG10 and rLaSota encoding the different $\mathrm{HN}$ proteins were used to recover the recombinant viruses.

Virus rescue was performed as described previously (Liu M.M. et al., 2015; Yu et al., 2017). Briefly, the respective helper plasmids individually encoding the NP, $\mathrm{P}$ and L proteins of either rSG10 or rLaSota were used along with their respective full-length cDNAs in transfected BSR T7 cells. Cells transfected with rLaSota chimeric cDNAs were cultured in medium with $5 \mu \mathrm{g} / \mathrm{ml} N$-tosylphenylalanine chloromethyl ketone (TPCK)-treated trypsin (Sigma-Aldrich, St. Louis, MO, United States) (Madansky and Bratt, 1978). Four days after transfection, the cell culture supernatant was inoculated into 9-day-old embryonated SPF chicken eggs via the allantoic cavity route. Recovery of the virus was confirmed by hemagglutination (HA) assay. Total RNA was extracted from NDV-positive allantoic fluid with TRIzol reagent (Invitrogen) according to the manufacturer's instructions. The sequences of the $\mathrm{HN}$ genes in the recovered chimeric viruses were confirmed by reverse transcription (RT) PCR and Western blot analysis.

\section{Construction of HN and F Protein Expression Plasmids}

The HN genes of strains BJ, LaSota, HB, Yucaipa and F gene of LaSota were amplified from full-length cDNA clones as described above and were inserted into pCI-neo (pCI) plasmid (Promega, Madison, WI, United States) between the EcoRI and XbaI restriction sites. The obtained plasmids were named pCI-BJHN, pCI-LaHN, pCI-HBHN, pCI-YuHN and pCI-LaF. The plasmids pCI-SGHN and pCI-SGF were generated in our laboratory as described previously (Jin et al., 2016).

\section{Western Blot Analysis}

Total protein lysates were extracted from infected or transfected cells with ice-cold RIPA lysis buffer ( $1 \%$ sodium deoxycholate, $1 \%$ Triton X-100, $50 \mathrm{mM}$ Tris [pH 7.4], $150 \mathrm{mM} \mathrm{NaCl}$ ). Cellular proteins were separated through $10 \%$ sodium dodecyl sulfate-polyacrylamide gel electrophoresis and transferred to a polyvinylidene difluoride (PVDF) membrane (Amersham Biosciences, Freiburg, Germany). Each PVDF membrane was blocked with 5\% (w/v) skim milk and $0.1 \%$ Tween 20 in Tris buffered saline (TBST) and then incubated with a primary antibody at $4^{\circ} \mathrm{C}$ overnight. Primary antibodies were SG10, Yucaipa polyclonal serum or anti-SG10 F rabbit polyclonal antiserum (both diluted 1:100), $\beta$-actin mouse monoclonal antibody with a dilution 1:1000 (Beyotime Biotechnology, Beijing, China). After being washed with TBST, the membranes were incubated with corresponding horseradish peroxidase (HRP)-conjugated anti-chicken, antirabbit or anti-mouse antibody at a 1:10,000 dilution for $1 \mathrm{~h}$ (Bioss Biotechnology, Beijing, China). Presence of HRP was detected using a Western Lightning chemiluminescence kit (CWBIO, Beijing, China). Protein bands were normalized to $\beta$-actin and quantified by densitometry using ImageJ software (National Institute of Mental Health, Bethesda, MD, United States).

\section{Virus Growth Kinetics}

The growth kinetics of nine NDVs, including the rSG10, rLaSota and their chimeric viruses were evaluated under multiplecycle growth conditions in DF-1 cells. Cells in duplicate wells of six-well plates were infected with NDVs at a multiplicity of infection (MOI) of 0.01 plaque forming units (PFU)/cell. After $1 \mathrm{~h}$ of adsorption, the cells were washed with DMEM and then incubated with DMEM containing 2\% FBS at $37^{\circ} \mathrm{C}$ in $5 \% \mathrm{CO}_{2}$. The media of cells infected with rLaSota and its chimeric viruses were supplemented with $5 \mu \mathrm{g} / \mathrm{ml}$ TPCK-treated trypsin. Supernatants were collected at $12 \mathrm{~h}$ intervals until $72 \mathrm{~h}$ post-infection (hpi), and the viral titers were quantified in DF-1 cells and expressed as median tissue culture infective doses $\left(\mathrm{TCID}_{50}\right) / \mathrm{ml}$, using the endpoint method (Reed and Muench, 1938). Cells infected with rLaSota and its chimeric viruses were identified by indirect immunofluorescence assay (IFA). In brief, at $72 \mathrm{hpi}$, cells were fixed with cold methanol for $20 \mathrm{~min}$ and incubated with anti-LaSota serum at $37^{\circ} \mathrm{C}$ for $1 \mathrm{~h}$. Then the cells were washed three times with phosphate-buffered saline (PBS) and goat anti-chicken IgG-FITC (Sigma-Aldrich) was added to each well. Plates were incubated at $37^{\circ} \mathrm{C}$ for $1 \mathrm{~h}$ and washed three times with PBS. Plates were examined on an inverted fluorescence microscope. 


\section{Virulence of Recovered Viruses}

The virulence of rSG10, rLaSota and their chimeric viruses was determined with standard virulence tests for NDV: the mean death time (MDT) in 9-day-old SPF embryonated chicken eggs, the intracerebral pathogenicity index (ICPI) in 1-day-old chicks and the intravenous pathogenicity index (IVPI) in 6-weekold chickens. All tests were performed according to previously published methods (Khattar et al., 2009).

\section{Viral Pathogenicity in Chickens}

To evaluate the pathogenicity of the rSG10-YuHN, rLaSotaSGHN and their parent strains (rSG10 and rLaSota), groups of 20 (10 for sampling and 10 for clinical observation) 3-weekold SPF chickens were inoculated with $10^{5} 50 \%$ egg infectious dose $\left(\mathrm{EID}_{50}\right)$ of virus per bird by the oculonasal route. The birds were observed daily and scored as follows for clinical signs for 14 days post-infection (dpi): 0 , healthy; 1 , sick; 2 , wing drop/paralysis/torticollis/incoordination; 3, prostration; 4, dead. Survival was monitored until $14 \mathrm{dpi}$. Two birds from each group were euthanized at 1,3,5,7 dpi, and brain, trachea, lung, spleen, duodenum and caecum were collected for virus titration. Samples collected at $3 \mathrm{dpi}$ were fixed in $10 \%$ buffered formalin for histopathology. For virus titration, tissue samples were homogenized in PBS containing antibiotics and the supernatant was serially diluted 10-fold and inoculated in DF-1 cells, in duplicate wells per dilution. The virus titers were determined by IFA as described above and the TCID 50 per $100 \mu \mathrm{g}$ of tissue was calculated (Reed and Muench, 1938). For histopathology, the fixed tissues were routinely embedded in paraffin wax, $5 \mu \mathrm{m}$ sections were prepared for hematoxylin and eosin staining and examined for lesions using light microscopy.

\section{Hemadsorption (HAd) Assay}

Confluent monolayers of Vero cells in 24-well plates were infected with $0.1 \mathrm{MOI}$ of virus. Cells were washed with cold PBS at $24 \mathrm{hpi}$ and then overlaid with a $2 \%(\mathrm{v} / \mathrm{v})$ suspension of CRBCs at $4^{\circ} \mathrm{C}$ for $30 \mathrm{~min}$ (Li et al., 2004). Unbound CRBCs were washed with ice-cold PBS and the CRBCs bound to the virus-infected cells were lysed with an RBC lysis solution ( $17 \mathrm{mM}$ Tris-HCl, $0.145 \mathrm{M} \mathrm{NH}_{4} \mathrm{Cl}$ ). The released hemoglobin was measured with a Spectramax M5 ELISA reader at $549 \mathrm{~nm}$ (Molecular Devices, Sunnyvale, CA, United States). The HAd assay investigating HN at the protein level was performed with monolayers of Vero cells transfected using $0.5 \mu \mathrm{g}$ each of pCI-HN plasmids (pCISGHN, pCI-BJHN, pCI-LaHN, pCI-HBHN and pCI-YuHN) as described previously.

\section{NA Assay}

The NA activity was determined using a modification of an assay described previously (Potier et al., 1979). Vero cell monolayers were infected with NDVs at $0.1 \mathrm{MOI}$. At $24 \mathrm{hpi}$, the cells were incubated at $37^{\circ} \mathrm{C}$ for $30 \mathrm{~min}$ with $30 \mu \mathrm{l}$ of substrate mix (one volume of $325 \mathrm{mM} 2-\mathrm{N}$-morpholinoethanesulfonic acid [MES; $\mathrm{pH}$ 6.4], two volumes of $0.5 \mathrm{mM} 2^{\prime}$-(4-methylumbelliferyl)- $\alpha$-D$\mathrm{N}$-acetylneuraminic acid [MUN; Sigma], and three volumes of $10 \mathrm{mM}$ calcium chloride) per well. The reaction was terminated using $0.014 \mathrm{M}$ sodium hydroxide in $83 \%(\mathrm{v} / \mathrm{v})$ ethanol. Fluorescence intensity was measured at an emission wavelength of $450 \mathrm{~nm}$ and an excitation wavelength of $360 \mathrm{~nm}$ with a Spectramax M5 ELISA reader (Molecular Devices). The NA assay investigating $\mathrm{HN}$ at the protein level was performed with monolayers of Vero cells transfected with the same expression plasmids utilized in HAd assay.

\section{Fusion Index Assay}

The fusogenic abilities of the rSG10 and its chimeric viruses were examined in Vero cells (Kim et al., 2011). Six-well plates seeded a day earlier at $2.0 \times 10^{6}$ cells were infected with NDVs at $0.1 \mathrm{MOI}$ and maintained in DMEM with $2 \% \mathrm{FBS}$ at $37^{\circ} \mathrm{C}$ under $5 \% \mathrm{CO}_{2}$. After monitoring cytopathic effect for $48-72 \mathrm{~h}$, the cells were washed once with $0.02 \%$ EDTA, and subsequently incubated for 2 min with $1 \mathrm{ml}$ of EDTA at room temperature. The cells were washed with PBS and fixed for 20 min with methanol at room temperature, and then stained with hematoxylin-eosin. Fusion was quantitated by the fusion index, i.e., the ratio of the total number of nuclei to the number of cells in which these nuclei were observed. The fusogenic properties of rLaSota and its chimeric viruses were examined in DF- 1 cells and the medium was supplemented with $5 \mu \mathrm{g} / \mathrm{ml}$ TPCK-treated trypsin. The fusion index assay at the protein level was performed with monolayers of Vero cells co-transfected using $1 \mu \mathrm{g}$ each of pCI$\mathrm{HN}$ and pCI-SGF. Co-transfection of pCI-HN and pCI-LaF was performed in DF-1 cells with $5 \mu \mathrm{g} / \mathrm{ml}$ TPCK-treated trypsin. The HAd, NA and fusion index values for all the viruses or HN proteins were expressed as percentages of the values for the parental viruses (rSG10, rLaSota) or the expressed $\mathrm{HN}$ and F protein (pCI-SGHN, pCI-SGHN/SGF, pCI-LaHN/LaF), which were considered to be $100 \%$.

\section{Hemolytic Assessment}

The hemolytic activities were performed as described previously (Bratt and Clavell, 1972). Briefly, the allantoic fluids of the rescued viruses were centrifuged with $500 \times \mathrm{g}$ for $20 \mathrm{~min}$, and the supernatants were diluted to the same hemagglutination unit $/ \mathrm{ml}$ (HAU/ml). Five hundred microliters of the diluted virus sample was mixed with $1 \mathrm{ml}$ of $1 \%$ CRBC suspension and incubated on ice for $20 \mathrm{~min}$. Then the CRBCs were centrifuged at $500 \times g$ for $3 \mathrm{~min}$, washed and resuspended with $0.5 \mathrm{ml}$ PBS. After $1 \mathrm{~h}$ incubation at $37^{\circ} \mathrm{C}$, tubes were centrifuged for $5 \mathrm{~min}$ at $200 \times g$. Subsequently, supernatant fluids were added to 96 -well plates and the hemoglobin contents were measured at $549 \mathrm{~nm}$ with a Spectramax M5 ELISA reader (Molecular Devices). Positive and negative controls were $0.03 \mathrm{M} \mathrm{NH}_{4} \mathrm{OH}$ and PBS, respectively. The hemolytic values for each virus were expressed as percentages of the values for parental virus rSG10 and rLaSota at $2^{8} \mathrm{HAU} / \mathrm{ml}$, which were considered to be $100 \%$.

\section{Data Analysis}

All data was analyzed using Prism 6.0 (GraphPad Software Inc., San Diego, CA, United States). Statistical differences among different groups were performed using the analysis of variance method followed by Tukey's test. Statistical significance was set at ${ }^{*} p<0.05,{ }^{* *} p<0.01$, and ${ }^{* * *} p<0.001$. 


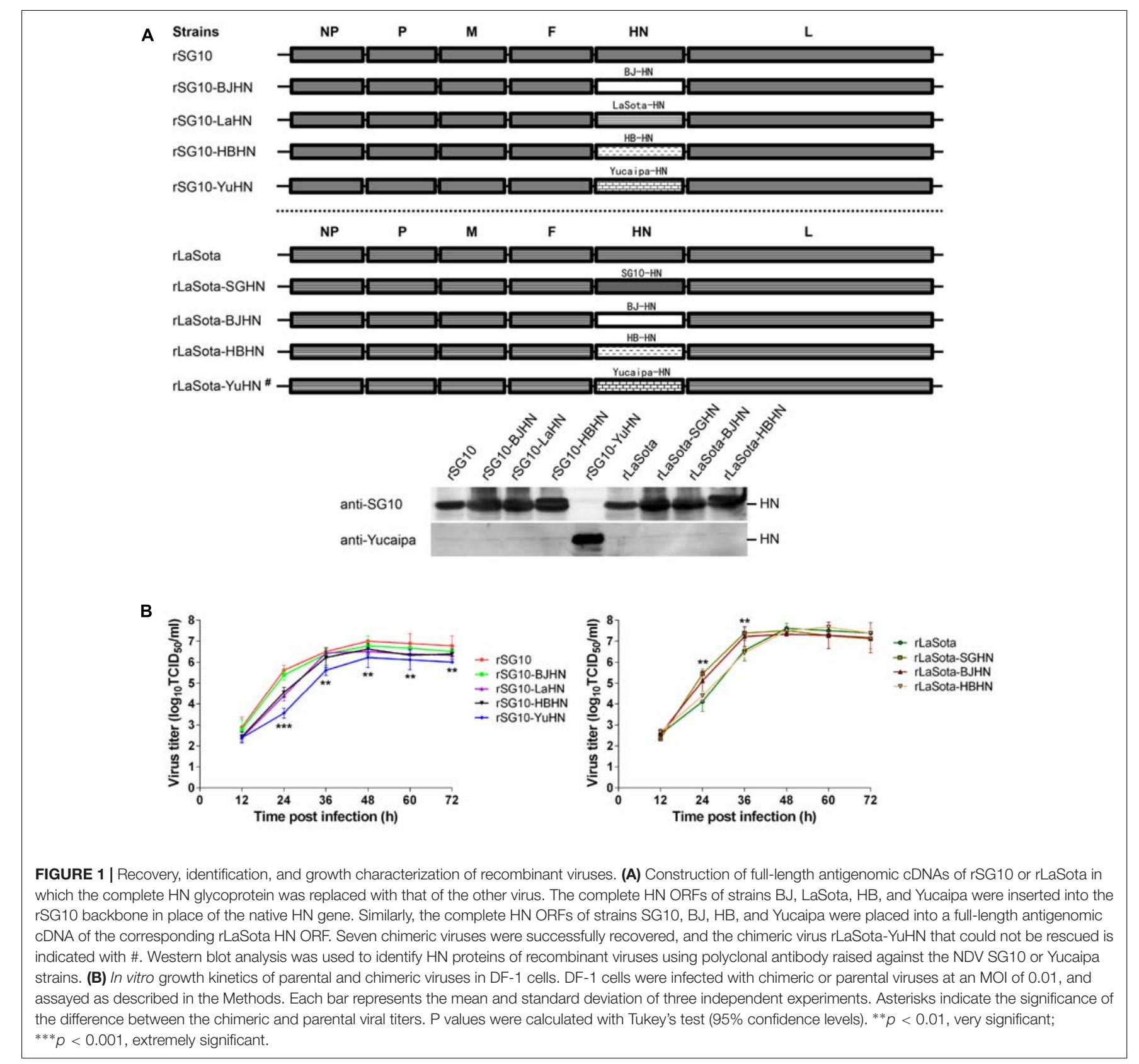

\section{RESULTS}

\section{Recovery and Identification of Recombinant Chimeric Viruses}

To investigate the impact of the different origins of HN proteins in NDV virulence, the complete HN ORF of strains BJ, LaSota, $\mathrm{HB}$ and Yucaipa was inserted into the rSG10 backbone in place of the corresponding rSG10 sequence. Likewise, the complete HN ORF of rLaSota was replaced with the corresponding ORF of strains SG10, BJ, HB and Yucaipa. The supernatants from transfected BSR T7/5 cells were passaged twice in embryonated SPF chicken eggs. The HA positive allantoic fluids were used for the isolation of viral RNA, followed by sequence analysis of an RT-PCR fragment to ensure the presence of the intended HN gene. Seven chimeric viruses were recovered, but the full-length cDNA encoding the Yucaipa HN protein in the rLaSota backbone did not yield a viable virus (Figure 1A). The rescued viruses were successively passaged 10 times via 9-day-old SPF eggs to further determine their stability. The sequencing results of the whole $\mathrm{HN}$ genes indicated that the seven rescued viruses were stable. Therefore, these seven chimeric viruses of the $10^{\text {th }}$ generations, together with their parental viruses (rSG10 and rLaSota), were used in subsequent experiments. The HN proteins in parental and chimeric viruses were analyzed by Western blotting using 
polyclonal anti-SG10 and anti-Yucaipa serum. This confirmed that the correct $\mathrm{HN}$ protein was present (Figure 1A).

\section{Growth of Chimeric Viruses in DF-1 Cells}

The multicycle replication of chimeric and parental viruses was evaluated in DF1 cells (Figure 1B). The rSG10 constructs bearing the HN genes of strains BJ, LaSota, HB and Yucaipa showed decreased replication compared with parental rSG10. Differences in the growth kinetics of the viruses were observed after $24 \mathrm{hpi}$. The replication kinetics of rSG10-BJHN were slightly lower than rSG10, whereas rSG10-YuHN showed significantly decreased viral yields than the parental virus ( $p<0.001$ at $24 \mathrm{hpi} ; p<0.01$ at 36-72 hpi). rSG10-LaHN and rSG10-HBHN replicated to titers that were intermediate between the extremes of rSG10 and rSG10-YuHN.

When the growth of strains rLaSota-SGHN, rLaSota-BJHN, rLaSota-HBHN was compared with that of the parental rLaSota, rLaSota-SGHN and rLaSota-BJHN showed an obvious increase at 24 and $36 \mathrm{hpi}(p<0.05)$. rLaSota and its chimeric viruses grew to similar titers after $32 \mathrm{hpi}$. These results show the relevance of $\mathrm{HN}$ to virus growth in vitro, indicating that the $\mathrm{HN}$ protein could affect the growth of NDV, and the origin of the HN protein has a discernible impact.

\section{Virulence Assessment of Chimeric Viruses}

Virulence of the seven chimeric viruses along with their respective parental viruses was evaluated by the MDT, the ICPI and the IVPI tests. The MDT values of rSG10 and rLaSota were $42 \mathrm{~h}$ and $>90 \mathrm{~h}$, and these viruses had ICPI scores of 1.93 and 0.00 , and IVPI values of 2.79 and 0.00 , respectively (Table 1 ). The chimeric viruses on the rSG10 background resulted in decreased virulence compared with parental rSG10. The MDT/ICPI/IVPI

TABLE 1 | Pathogenicity of the parental and recombinant viruses.

\begin{tabular}{lccc}
\hline Virus & MDT $(\mathbf{h})^{\mathbf{a}}$ & ICPI score $^{\mathbf{b}}$ & IVPI score $^{\mathbf{c}}$ \\
\hline rSG10 & 42.0 & 1.93 & 2.79 \\
rSG10-BJHN & 50.4 & 1.86 & 2.67 \\
rSG10-LaHN & 57.6 & 1.76 & 2.38 \\
rSG10-HBHN & 55.2 & 1.81 & 2.64 \\
rSG10-YuHN & 72.0 & 1.58 & 1.91 \\
rLaSota & $>90$ & 0.00 & 0.00 \\
rLaSota-SGHN & $>90$ & 0.63 & 0.58 \\
rLaSota-BJHN & $>90$ & 0.51 & 0.41 \\
rLaSota-HBHN & $>90$ & 0.13 & 0.20 \\
\hline
\end{tabular}

aThe minimum lethal dose of virus required to kill all the embryonated chicken eggs inoculated with virus by the allantoic route. Mean death time (MDT) pathotype definition: virulent strains, $<60 \mathrm{~h}$; moderately virulent strains, 60-90 h; avirulent strains, $>90 \mathrm{~h}$. ${ }^{\mathrm{b}}$ Determined by inoculating groups of 10 one-day-old SPF chicks with fresh infective allantoic fluid via the intracerebral route. Intracerebral pathogenicity index (ICPI) definition: virulent strains, 1.5-2.0; moderately virulent strains, 0.7-1.5; avirulent strains, 0.0-0.7. ${ }^{\mathrm{C} D e t e r m i n e d ~ b y ~ i n o c u l a t i n g ~ g r o u p s ~ o f ~}$ 10 six-week-old SPF chickens with fresh infective allantoic fluid via the intravenous route. Intravenous pathogenicity index (IVPI) definition: velogenic strains yield values greater than 2.0, while avirulent strains approach 0.0. values were as follows: rSG10-BJHN, 50.4h/1.86/2.67; rSG10LaHN, 57.6h/1.76/2.38; rSG10-HBHN, 55.2h/1.81/2.64; rSG10YuHN, 72h/1.58/1.91, respectively. These results show that transfer of the Yucaipa HN protein into rSG10 had the greatest effect on decreasing virulence, altering the viral pathotype from velogenic to borderline mesogenic.

Chimeric viruses on the rLaSota background showed increased virulence compared with parental rLaSota. The MDT/ICPI/IVPI values were as follows: rLaSota-SGHN, $>90 \mathrm{~h} / 0.63 / 0.58$; rLaSota-BJHN, $>90 \mathrm{~h} / 0.51 / 0.41$; rLaSotaHBHN, $>90 \mathrm{~h} / 0.13 / 0.20$, respectively (Table 1 ). These findings show that transfer of the SG10 HN protein into rLaSota had the greatest effect on increasing virulence, resulting in a change in viral pathotype from lentogenic to borderline mesogenic. Thus, in both series, the virulence was affected by transfer of the HN gene.

\section{Replication and Pathogenicity of Chimeric Viruses in 3-week-old SPF Chickens}

To further evaluate the pathogenicity of two chimeric viruses (rSG10-YuHN and rLaSota-SGHN) and their parental viruses (rSG10 and rLaSota), 3-week-old chickens were inoculated with $10^{5} \mathrm{EID}_{50}$ of virus per bird via the oculonasal route. All chickens infected with the parental rSG10 virus showed clinical signs at 3 dpi and $100 \%$ mortality at 4 dpi. At $5 \mathrm{dpi}$, clinical signs were observed in chickens infected with rSG10YuHN. One chicken was found dead in the rSG10-YuHN group at both 8 and 9 dpi. All chickens infected with rSG10YuHN began to recover at $10 \mathrm{dpi}$, and the clinical score was much lower than with rSG10. No obvious clinical signs were observed in any birds inoculated with rLaSota and rLaSotaSGHN (Figures 2A,B).

At $1,3,5$, and $7 \mathrm{dpi}$, two chickens from each group were sacrificed and tissue samples were collected for virus titration (Figure 2C). For rSG10 and rSG10-YuHN, all sampled tissues in the rSG10-infected chickens showed high viral titers at $3 \mathrm{dpi}$. However, the virus titers in trachea, lung, spleen, duodenum and caecum were significantly lower in rSG10YuHN-infected chickens than in chickens inoculated with rSG10 ( $p<0.001)$, indicating a decreased replication ability of rSG10-YuHN compared with the parental virus. For rLaSota and rLaSota-SGHN, rLaSota could replicate in trachea and occasionally in lung, spleen and caecum, while rLaSota-SGHN showed an increased replication ability in a wide range of organs. rLaSota-SGHN replicated to moderate or high titers in trachea, lung, spleen and caecum, and showed significant differences ( $p<0.001$ or $p<0.01$ for individual organs) compared with rLaSota.

Two chickens from each group were sacrificed at $3 \mathrm{dpi}$ for histopathological analysis (Figure 3). The rSG10 caused the moderate to severe histological changes in the sampled tissues: loose brain tissue with vacuolation (empty arrows); interstitial broadening, edema (black arrow) in the trachea; bronchiectasis and emphysema (black arrow) in the lung; excessive inflammatory cell infiltration, multifocal confluent 

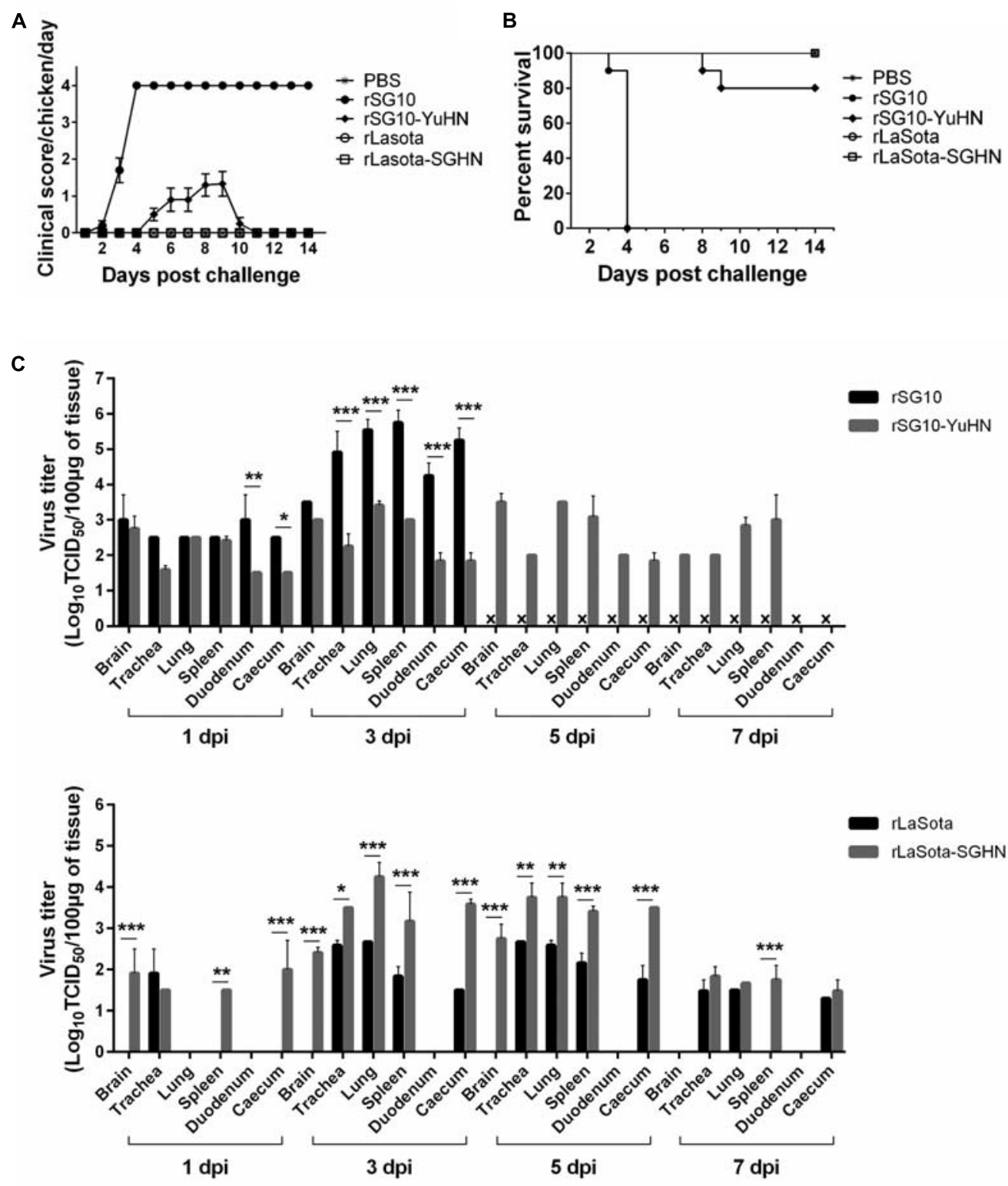

FIGURE 2 | Clinical scores, survival rate, and replication of parental and chimeric viruses in 3-week-old chickens. Groups of 20 (10 for sampling and 10 for clinical observation) 3-week-old SPF chickens were inoculated with $10^{5}$ EID 50 of rSG10, rSG10-YuHN, rLaSota, and rLaSota-SGHN through the natural route of infection. (A) Clinical signs in infected chickens were scored daily based on 10 birds per group (0, healthy; 1, sick; 2, wing drop/paralysis/torticollis/incoordination; 3, prostration; 4, dead). The daily mean scores for per group are shown. (B) Survival of 3-week-old SPF chickens inoculated with the parental and chimeric viruses based on 10 birds per group. (C) Replication of parental and chimeric viruses in 3-week-old chickens. The inoculated birds were sacrificed at 1, 3, 5, 7 dpi, the indicated tissues collected and virus titers determined by indirect immunofluorescence in DF-1 cells. The symbol " $\times$ " indicates no chickens survived for detection of virus. Asterisks indicate the significance of the difference between the chimeric and parental viral titers. ${ }^{*} p<0.05$, significant; ${ }^{* *} p<0.01$, very significant; $* * * p<0.001$, extremely significant.

coagulative necrosis (empty arrow) and lymphocyte necrosis (black arrow) in the spleen; mucosal epithelium exfoliation (black arrow) in the duodenum; mucosal epithelium exfoliation (black arrow), increased goblet cells in the mucosal epithelium (empty arrow) of the caecum. However, the chimera rSG10-YuHN exhibited decreased virulence with mild lesions in the trachea and lung: interstitial broadening, edema (black arrow) and slight exfoliation of the tracheal mucosa epithelium (empty arrow), bronchiectasis and emphysema (black arrow) in the lung, but no obvious changes in the brain, spleen, duodenum or caecum compared with the parental rSG10 virus.

For the chimera rLaSota-SGHN and its parental virus rLaSota, no obvious histological changes were found in sampled tissues 


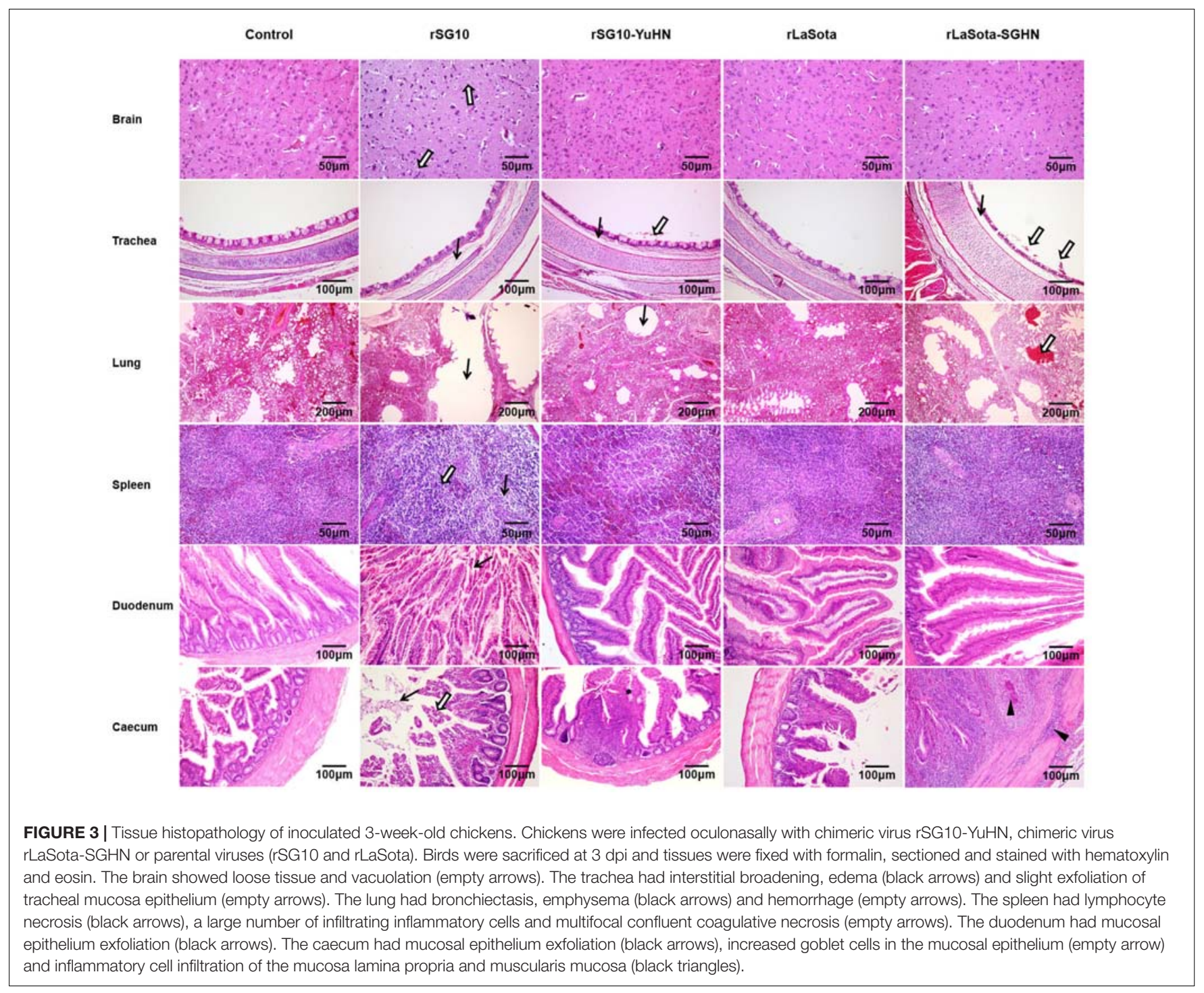

of the group inoculated with rLaSota. On the contrary, rLaSotaSGHN caused increased virulence with mild or moderate histological changes in trachea, lung and caecum: interstitial broadening, edema (black arrow) and slight exfoliation of the tracheal mucosa epithelium (empty arrows), hemorrhage (empty arrow) in the lung, and inflammatory cell infiltration to the mucosa lamina propria and muscularis mucosa (black triangles) in the caecum (Figure 3). These results demonstrate that the $\mathrm{HN}$ protein affects replication and pathogenicity of NDV in chickens, and the extent of the impact is related to the origin of the $\mathrm{HN}$ protein.

\section{HAd and NA Activities of Chimeric Viruses}

To determine whether the different origins of HN proteins have a differing influence on the biological activities of NDV in cultured cells, the mutant and parental viruses were determined for their HAd and NA activities (Figure 4). The HN protein expression of recombinant viruses was measured in Vero cells, and indicated that $\mathrm{HN}$ expression in the chimeric viruses was similar to that of the respective parental HN (Figures 4A,D). The biological activities of each chimeric virus were analyzed and calculated as a percentage of that of the respective parental virus (rSG10 and rLaSota), whose biological activities were deemed to be $100 \%$. Figures $4 B, C$ show that HAd and NA activities of rSG10BJHN were slightly lower than the parental virus rSG10, whereas significant reductions were observed in both HAd and NA activities for chimeras rSG10-LaHN (78 and 76\%, respectively; $p<0.01$ ), rSG10-HBHN (77 and 77\%, respectively; $p<0.01$ ), and rSG10-YuHN (63 and 65\%, respectively; $p<0.001$ ). For the chimeric viruses with the rLaSota backbone, rLaSota-SGHN and rLaSota-BJHN showed a 56 and $42 \%$ increase, respectively, in HAd activity, and 40 and $28 \%$ increase, respectively, in NA activity over those of the rLaSota parent $(p<0.001)$. HAd and NA activities of rLaSota-HBHN were similar to that of the parent virus (Figures 4E,F). These results reiterate the importance of the $\mathrm{HN}$ protein in the attachment and NA functions of NDV in the 

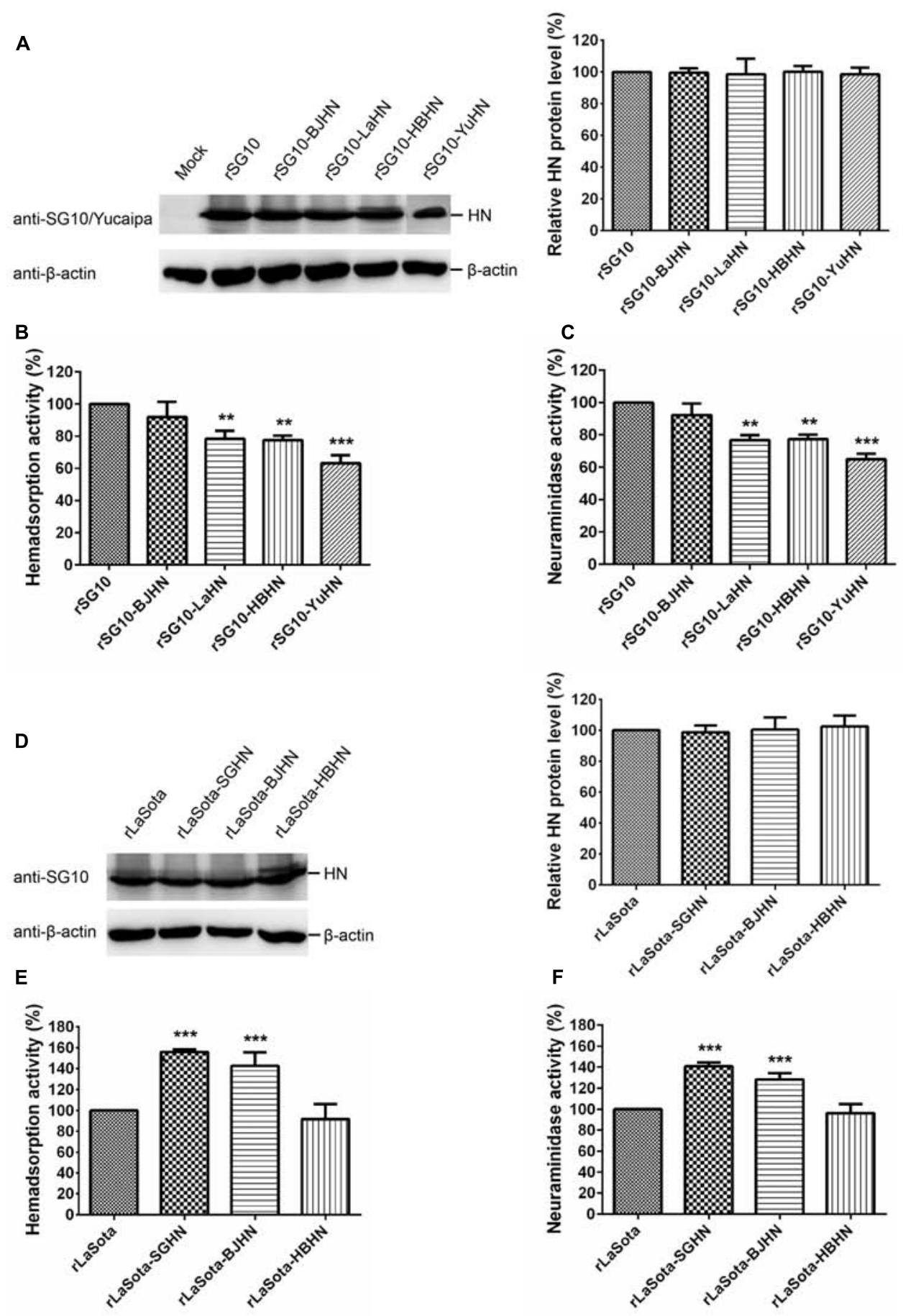

FIGURE 4 | HAd and NA activities of parental and chimeric viruses. (A,D) The expression amount of each HN protein was determined by Western blotting using anti-SG10 or anti-Yucaipa serum. HAd (B,E) and NA activities (C,F) were examined in Vero cells infected with virus at an MOI of 0.1. HN protein expression level, HAd and NA activities of these viruses are expressed as percentages of the values for parental viruses (rSG10 and rLaSota), which were set at 100\%. Each bar represents the mean and standard deviation of three independent experiments. Asterisks indicate the significance of the differences between the biological activity of a chimeric virus and that of the parental virus. P values were calculated with Tukey's test $\left(95 \%\right.$ confidence levels). ${ }^{* *} p<0.01$, very significant; ${ }^{* * *} p<0.001$, extremely significant. 
A

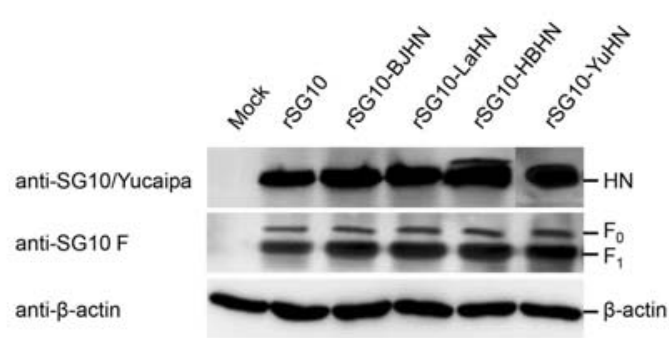

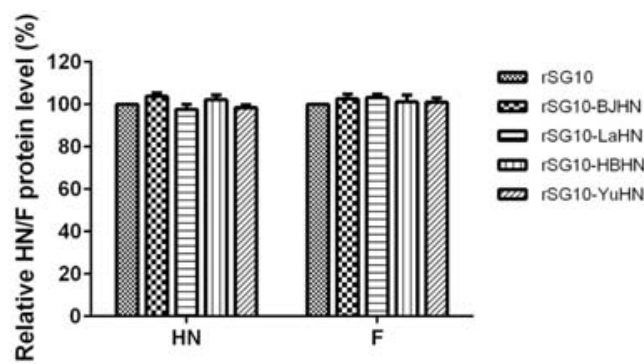

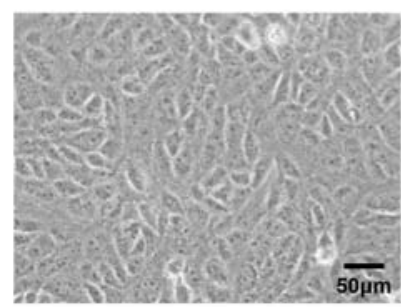

Mock

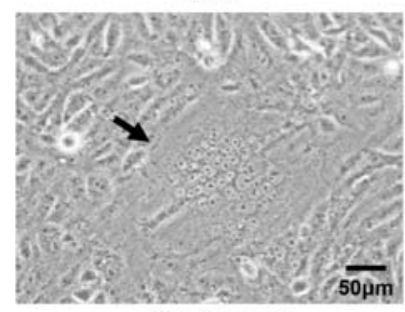

rSG10-LaHN

C

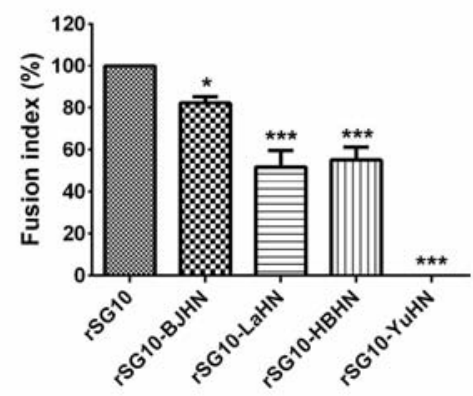

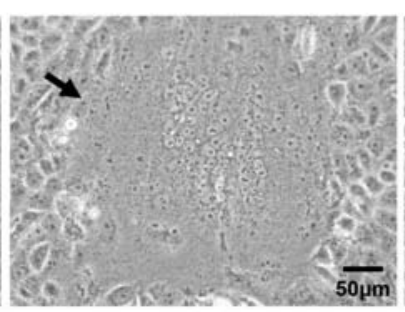

rSG10

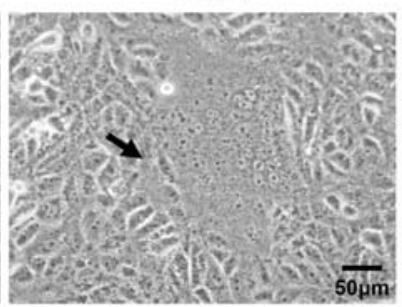

rSG10-HBHN

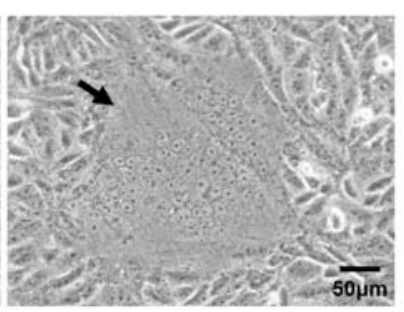

rSG10-BJHN

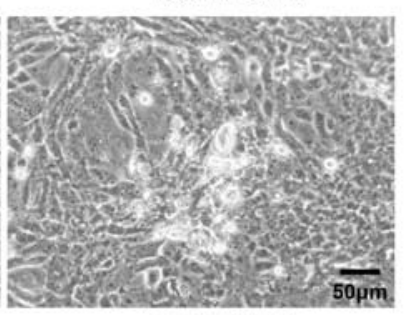

rSG10-YuHN
D

FIGURE 5 | Syncytium formation and hemolytic activities of $r S G 10$ and its chimeric viruses. (A) The levels of each $\mathrm{HN}_{\mathrm{N}}$ and $\mathrm{F}\left(\mathrm{F}_{0}\right.$ and $\left.\mathrm{F}_{1}\right)$ proteins were quantitated by Western blotting using sera specific to the individual SG10, Yucaipa or anti-SG10 F protein rabbit polyclonal antiserum, as indicated. The expression of HN and $\mathrm{F}$ proteins are expressed as percentages of the values for rSG10. (B) Syncytium formation was induced by viral infection of Vero cells. Black arrows indicate syncytia. Bar indicates $50 \mu \mathrm{m}$. (C) The fusion index values of chimeric viruses were calculated as the ratio of the total number of nuclei to the number of cells in which the nuclei were observed and expressed relative to the value for rSG10 (100\%). (D) The hemolytic activities for chimeric viruses were expressed as percentages of the value for rSG10 at $2^{8} \mathrm{HAU} / \mathrm{ml}$, which was set at $100 \%$. Each bar represents the mean and standard deviation of three independent experiments. Asterisks indicate statistically significant differences. P-values were calculated with Tukey's test ( $95 \%$ confidence levels). ${ }^{*} p<0.05$, significant; *** $p<0.001$, extremely significant.

context of viral infection. Furthermore, these data demonstrate that the different origins of HN proteins influence the HAd and NA activities of NDV to varying degrees.

\section{Fusion Indices and Hemolytic Activities of the Recombinant Viruses}

To further examine whether the substitution of the different HN proteins affected the fusogenic promotion activity of the virus, we measured fusion indices of rSG10 and its chimeric viruses in Vero cells, rLaSota and its chimeric viruses in DF-1 cells, and the hemolytic activities of all experimental viruses (Figures 5, 6). Western blot analysis indicated that the $\mathrm{HN}$ and $\mathrm{F}\left(\mathrm{F}_{0}\right.$ and $\mathrm{F}_{1}$ ) expressions of the derivatives of $\mathrm{rSG} 10$ and rLaSota were similar to that of respective parental virus (Figures 5A, 6A). The syncytia induced by rSG10-BJHN, rSG10-LaHN and rSG10HBHN were markedly smaller than those induced by rSG10, while syncytium was not induced by rSG10-YuHN (Figure 5B). 
A

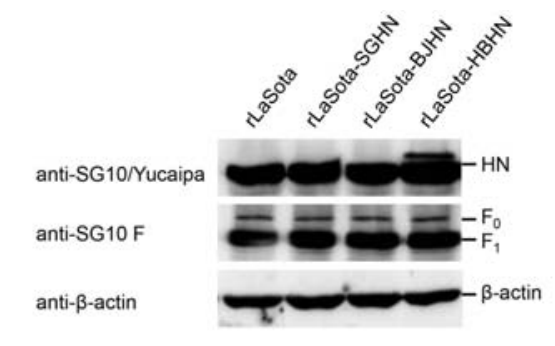

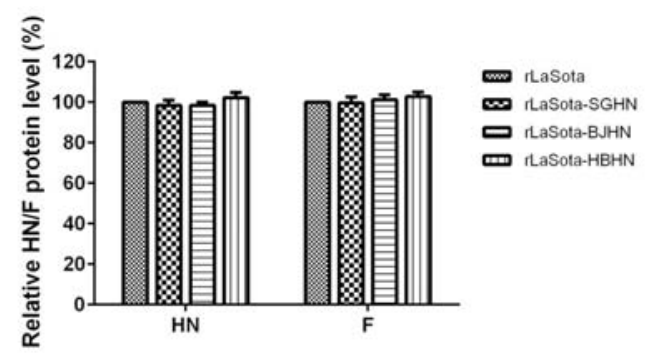

B
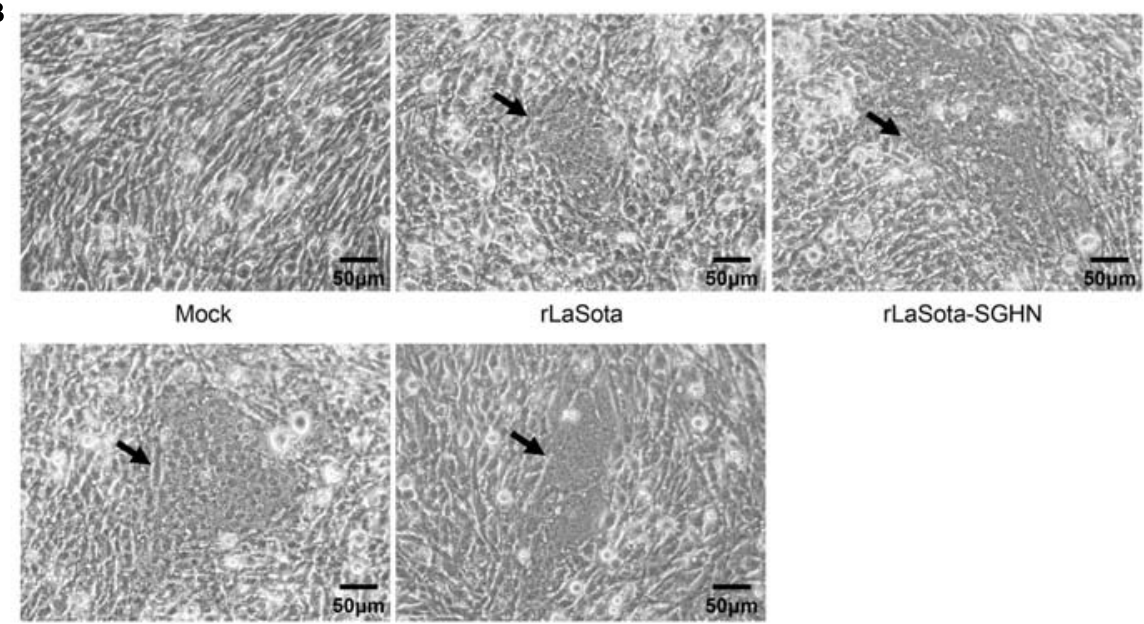

rLaSota-BJHN

C

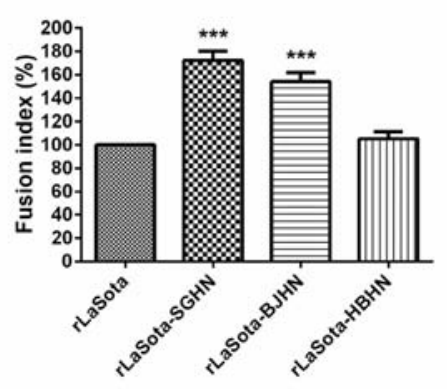

rLaSota

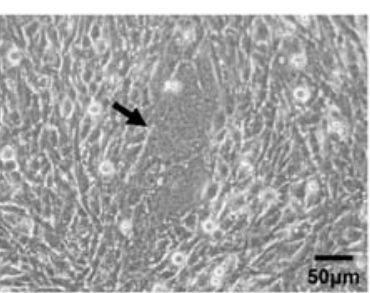

rLaSota-HBHN

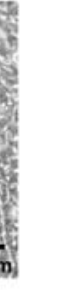

D

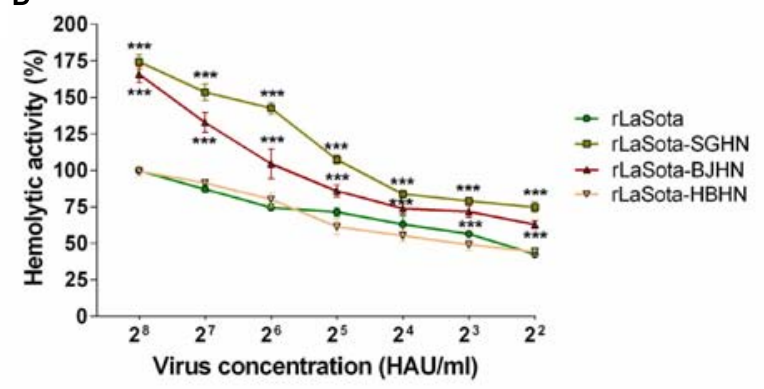

FIGURE 6 | Syncytium formation and hemolytic activities of rLaSota and its chimeric viruses. (A) The levels of each $H N$ and $F\left(F_{0}\right.$ and $\left.F_{1}\right)$ proteins were quantitated by Western blotting using sera specific to the individual SG10, Yucaipa and anti-SG10 F protein rabbit polyclonal antiserum, as indicated. The expression of HN and F proteins are expressed as percentages of the values for rLaSota. (B) Syncytium formation was induced by viral infection of DF-1 cells with $5 \mu \mathrm{g} / \mathrm{ml}$ TPCK-treated trypsin. Black arrows indicate syncytia. Bar indicates $50 \mu \mathrm{m}$. (C) The fusion index values of chimeric viruses were calculated as the ratio of the total number of nuclei to the number of cells in which the nuclei were observed and expressed relative to the value for rLaSota (100\%). (D) The hemolytic activities for chimeric viruses were expressed as percentages of the value for rLaSota at $2^{8} \mathrm{HAU} / \mathrm{ml}$, which was set at $100 \%$. Each bar represents the mean and standard deviation of three independent experiments. Asterisks indicate statistically significant differences. $P$-values were calculated with Tukey's test (95\% confidence levels). ${ }^{* * *} p<0.001$, extremely significant.

For the chimeric viruses with the rSG10 backbone, the fusion indices (relative to rSG10, as 100\%) were as follows: rSG10BJHN, 82\%; rSG10-LaHN, 52\%; rSG10-HBHN, 55\%; rSG10YuHN, 0\% (Figure 5C). Conversely, for the chimeric viruses with the rLaSota backbone, the size of syncytia induced by rLaSota-SGHN and rLaSota-BJHN were increased significantly compared with those induced by parental rLaSota (Figure 6B). The fusion indices (relative to rLaSota, as 100\%) were as follows: rLaSota-SGHN, 172\%; rLaSota-BJHN, 154\%; rLaSota-HBHN,
105\% (Figure 6C). These results showed that the origin of the HN protein had differing effects on the fusogenic promotion activity of the virus.

Subsequently, the hemolytic activities of recombinant viruses were tested at virus concentrations ranging from $2^{2}$ to $2^{8}$ $\mathrm{HAU} / \mathrm{ml}$. The hemolytic values are expressed as percentages of the values for parental viruses rSG10 and rLaSota at $2^{8} \mathrm{HAU} / \mathrm{ml}$, which were considered to be $100 \%$. For rSG10 derivatives bearing the HN genes of strains BJ, LaSota, HB and Yucaipa, 

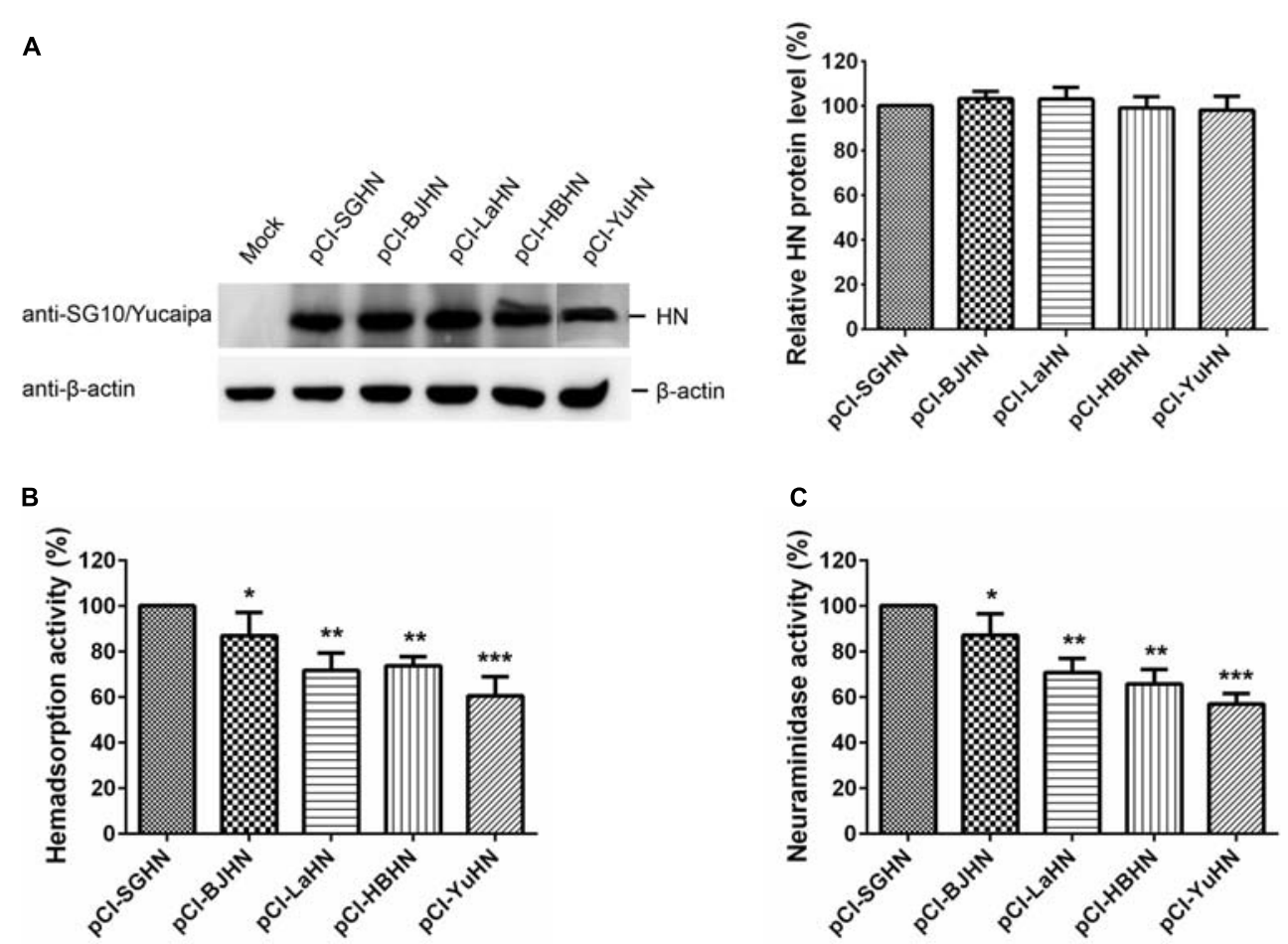

FIGURE 7 | HAd and NA activities of the different origins of HN proteins at the protein level. (A) The relative expression of each HN protein was examined by Western blot analysis at $24 \mathrm{~h}$ post-transfection of Vero cells by using anti-SG10 or anti-Yucaipa serum. HAd (B) and NA activities (C) were measured in Vero cells transfected with the $0.5 \mu \mathrm{g}$ each of $\mathrm{pCl}-\mathrm{HN}$ plasmids. The HN protein level, HAd and NA activities of the HN proteins of different origins are expressed as percentages of the values for expressed SGHN protein, which was considered to be 100\%. Each bar represents the mean and standard deviation of three independent experiments. Asterisks indicate statistically significant differences. ${ }^{*} p<0.05$, significant; ${ }^{*} p<0.01$, very significant; ${ }^{* * *} p<0.001$, extremely significant.

the hemolytic activity of rSG10-BJHN was slightly lower than parental virus rSG10, whereas rSG10-LaHN, rSG10-HBHN and rSG10-YuHN were significantly decreased compared with rSG10 $(p<0.001)$. Furthermore, rSG10-YuHN showed the greatest reduction in hemolytic activity of all the mutants (Figure 5D). As shown in Figure 6D, for rLaSota derivatives bearing the HN genes of strains SG10, BJ and HB, the hemolytic activity of rLaSota-HBHN was similar to that of rLaSota, but rLaSotaSGHN and rLaSota-BJHN were significantly increased compared with parental rLaSota $(p<0.001)$. These results indicate that the hemolytic activity of NDV is influenced by $\mathrm{HN}$, and the origin of this protein can influence hemolysis and fusion of the virus.

\section{Biological Activities of HN Proteins of Different Origins at the Protein Level}

Next, we investigated the HAd, NA and fusogenic promotion activities of the various $\mathrm{HN}$ proteins at the protein level, using HN expression plasmids (pCI-SGHN, pCI-BJHN, pCI-LaHN, pCI-HBHN and pCI-YuHN). The results were consistent with those of rSG10 derivatives bearing the HN genes of strains BJ, LaSota, HB and Yucaipa at the virus level; expression of the different HN proteins was similar in Vero cells (Figure 7A). In this case, BJHN, LaHN, HBHN and YuHN proteins showed an $87,72,74$, and $61 \%$ decrease, respectively, in HAd activity and
$87,71,66,57 \%$ decrease, respectively, in NA activity compared with the SGHN protein (Figures 7 B,C) $(p<0.05, p<0.01$, or $p<0.001$, depending on HN origin). These results demonstrate that HAd and NA activities of HN proteins of different origins varied at the protein level, with SGHN showing the highest HAd and NA activities, while receptor-binding ability and NA activity of YuHN protein were lowest.

Furthermore, the fusogenic promotion activities at the protein level of the different $\mathrm{HN}$ proteins were evaluated in Vero (Figure 8) and DF-1 cells (Figure 9) using HN protein expression plasmids (pCI-SGHN, pCI-BJHN, pCI-LaHN, pCI-HBHN, and pCI-YuHN) and F protein expression plasmids (pCI-SGF and pCI-LaF). The result had the same trend as at the viral level. The HN proteins of different origins showed significantly decreased fusion indices compared with co-transfection of pCI-SGHN and pCI-SGF under similar $\mathrm{HN}$ and $\mathrm{F}$ expression. The order of reduction (relative to SGHN/SGF, 100\%) was BJHN/SGF (85\%; $p<0.05)$, LaHN/SGF (56\%; $p<0.001)$, HBHN/SGF (51\%; $p<0.001)$ and YuHN/SGF (0\%; $p<0.001$ ) (Figure 8C). As for the co-expression of the different $\mathrm{HN}$ proteins and LaSota $\mathrm{F}$ protein (LaF) in DF-1 cells, syncytium formation of SGHN/LaF and $\mathrm{BJHN} / \mathrm{LaF}$ showed a 26 and $17 \%$ increase, respectively, over LaHN/LaF $(p<0.001)$. The fusion index of HBHN/LaF was roughly identical to the LaHN/LaF value. Syncytia were not induced by co-expression of YuHN and LaF (Figures 9B,C). 

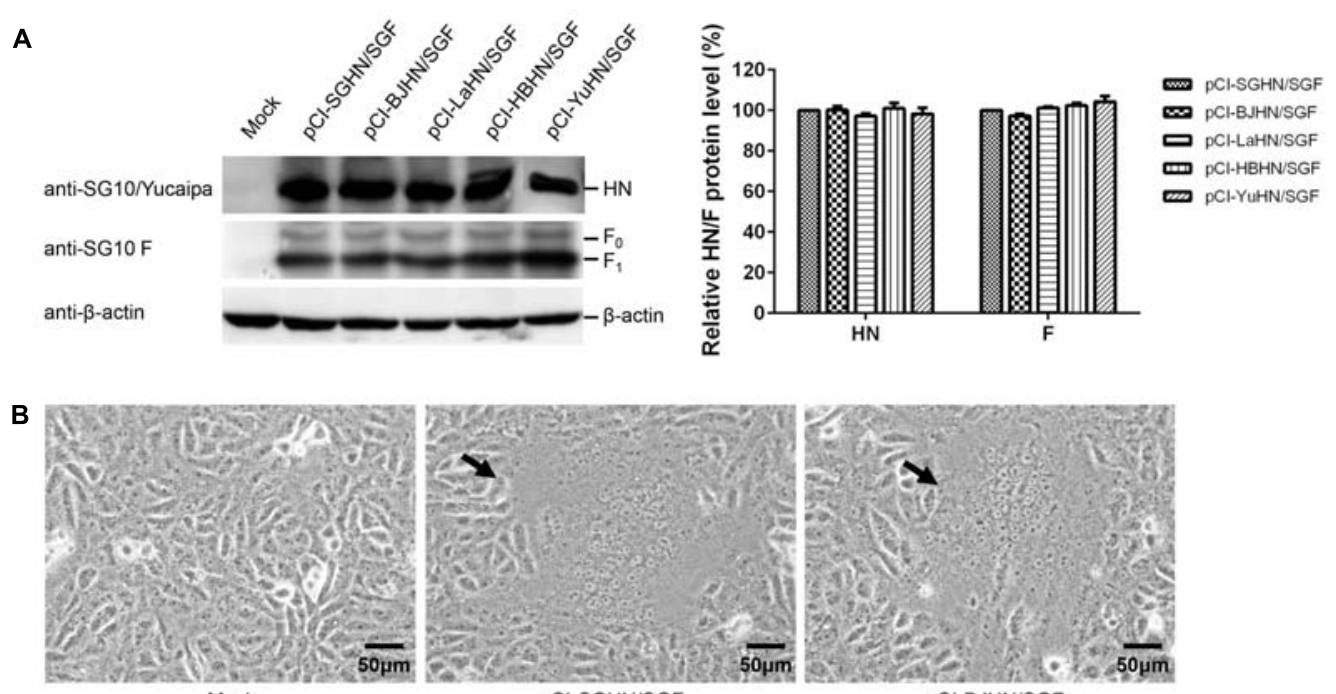

Mock pCI-SGHN/SGF

pCI-BJHN/SGF
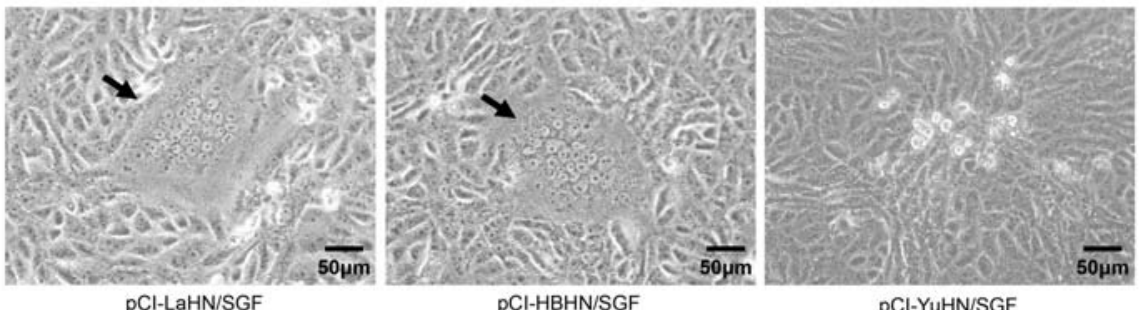

C

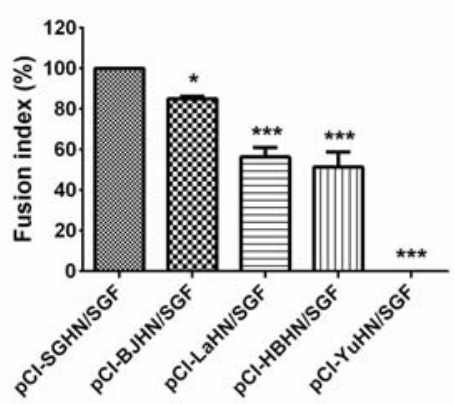

FIGURE 8 | Syncytium formation in Vero cells co-transfected with expressed plasmids. (A) The relative expression of each $\mathrm{HN}$ or $\mathrm{F}\left(\mathrm{F}_{0}\right.$ and $\left.\mathrm{F}_{1}\right)$ protein was measured by Western blotting using sera specific to the individual SG10, Yucaipa and anti-SG10 F protein rabbit polyclonal antiserum. (B) Syncytium formation was induced by co-transfection with $1 \mu \mathrm{g}$ each of $\mathrm{pCl}-\mathrm{HN}$ and $\mathrm{pCl}$-SGF. Black arrows indicate syncytia. Bar indicates 50 $\mu \mathrm{m}$. (C) The fusion index values were calculated as the ratio of the total number of nuclei to the number of cells in which the nuclei were observed. All values are expressed relative to the value for pCl-SGHN/SGF (100\%). Each bar represents the mean and standard deviation of three independent experiments. Asterisks indicate statistically significant differences. $P$ values were calculated with Tukey's test (95\% confidence levels). ${ }^{*} p<0.05$, significant; ${ }^{* * *} p<0.001$, extremely significant.

These results indicate that fusogenic promotion activities of the different HN proteins are different at the protein level, as SGHN protein showed the highest fusogenic promotion ability, while $\mathrm{YuHN}$ protein was unable to induce syncytium formation.

\section{DISCUSSION}

The virulence of different NDVs shows great variation within the single serotype and the molecular basis for this is not fully understood. Previous studies have shown that the amino acid sequence at the $\mathrm{F}$ protein cleavage site is a major determinant, but multiple proteins are associated with NDV virulence (Nagai et al., 1976; Toyoda et al., 1987; Glickman et al., 1988; Peeters et al., 1999; Huang et al., 2003; Panda et al., 2004; De Leeuw et al., 2005; Rout and Samal, 2008; Dortmans et al., 2010; Paldurai et al., 2014; Yu et al., 2017). In this study, exchange of the $\mathrm{HN}$ gene from a velogenic or lentogenic NDV backbone strain and other strains with different virulence was performed to reveal the exact role of the HN protein in NDV virulence. This is the first 

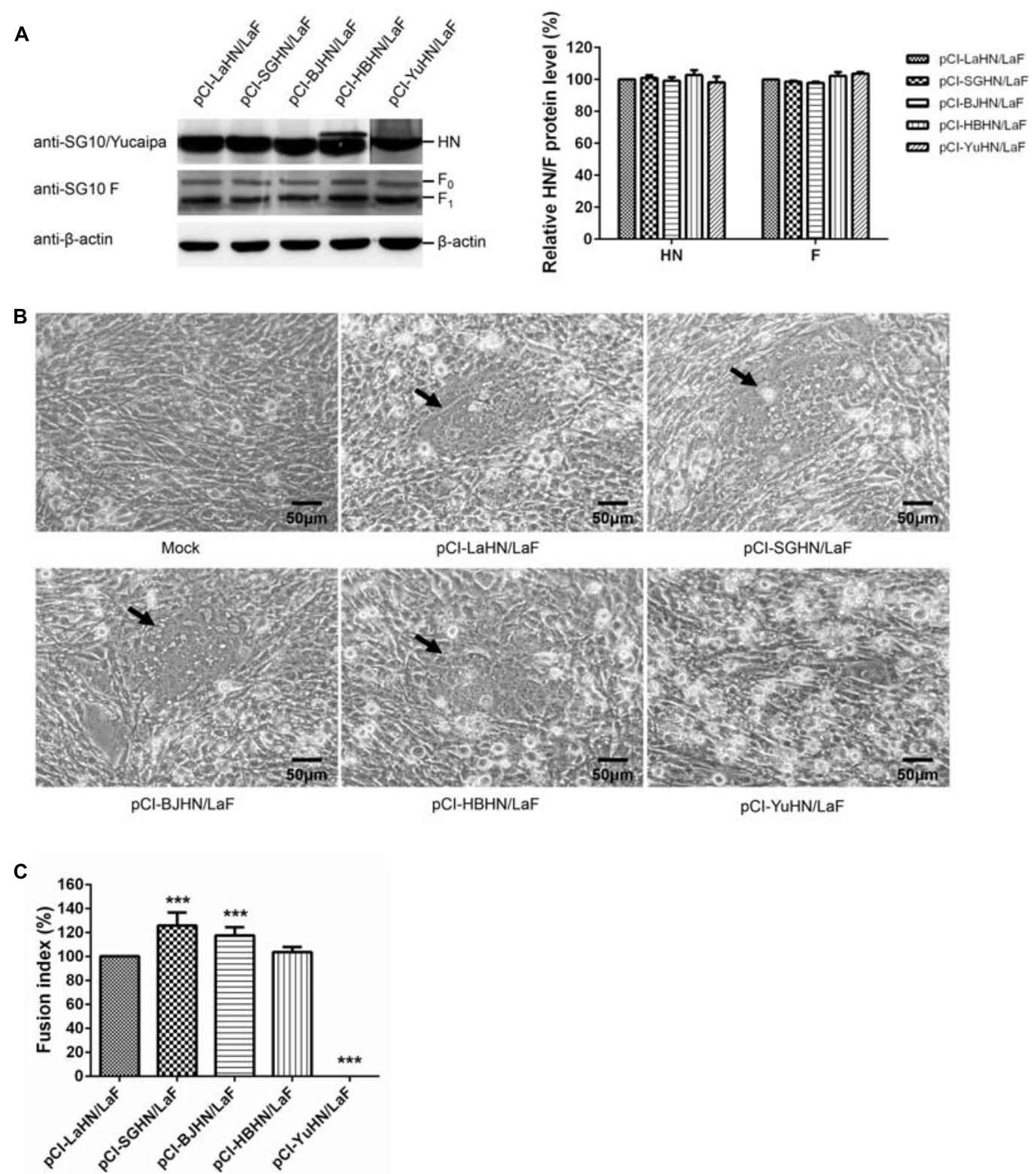

FIGURE 9 | Syncytium formation in DF-1 cells co-transfected with expressed plasmids. (A) The amount of each HN or $F\left(F_{0}\right.$ and $\left.F_{1}\right)$ protein expressed was examined by Western blot analysis using sera specific to the individual SG10, Yucaipa and anti-SG10 F protein rabbit polyclonal antiserum. (B) Syncytium formation was induced by co-transfection with $1 \mu \mathrm{g}$ each of $\mathrm{pCl}-\mathrm{HN}$ and pCl-LaF. Black arrows indicate syncytia. Bar indicates $50 \mu \mathrm{m}$. (C) The fusion index values were calculated as the ratio of the total number of nuclei to the number of cells in which the nuclei were observed. All values are expressed relative to the value for $\mathrm{pCl}-\mathrm{LaHN} / \mathrm{LaF}(100 \%)$. Each bar represents the mean and standard deviation of three independent experiments. Asterisks indicate statistically significant differences. $P$-values were calculated with Tukey's test (95\% confidence levels). ${ }^{* * *} p<0.001$, extremely significant.

comprehensive and systematic study of the HN protein and NDV virulence.

The HN protein of NDV plays multiple roles during host viral infection, including receptor binding, neuraminidase and fusion promotion activities (Crennell et al., 2000; Morrison, 2001; Melanson and Iorio, 2004). Some studies have indicated that the $\mathrm{HN}$ protein is also an important determinant of NDV virulence, but great variation exists between the results of different studies (Huang et al., 2004; De Leeuw et al., 2005; Kim et al., 2011; Adu-Gyamfi et al., 2016). Differences in these studies may be associated with the different NDV strains involved. Kim et al. (2011) investigated the role of the $\mathrm{F}$ and $\mathrm{HN}$ proteins by designing chimeric viruses in which the $\mathrm{F}$ and $\mathrm{HN}$ or their ectodomains were exchanged individually or together between the moderately virulent NDV strain Beaudette C and the avirulent APMV-2 strain Yucaipa. 
They recovered only three viruses: the recombinant APMV2 strain Yucaipa (rAPMV-2) which contained the NDV F protein, rAPMV-2 containing both the F and HN ectodomains of NDV, and rNDV which contained both the $\mathrm{F}$ and $\mathrm{HN}$ ectodomains of APMV-2. In this study, we successfully recovered the recombinant NDV strain rSG10, containing the APMV-2 Yucaipa HN glycoprotein in place of its own. The construct could be stably passaged despite a low amino acid sequence homology of $33.4 \%$ between NDV SG10 and APMV-2 Yucaipa, indicating that $\mathrm{HN}$ proteins have a level of compatibility among different APMVs. However, viable virus could not be recovered from the chimeric NDV LaSota cDNA that contained the APMV-2 Yucaipa HN gene after many attempts. The virulent strains with stronger infectivity tended to be favorable for viral rescue and this may explain the failure to recover avirulent chimeras.

Although disparities in tissue tropism and in vivo pathogenicity can be observed in some viruses, it is quite conceivable that viral tissue tropism is an important factor determining the virulence and broad tropism results in more severe pathogenicity or higher virulence for many viruses (Haller et al., 1995; Dortmans et al., 2011; Meliopoulos et al., 2014; Chhabra et al., 2016). For NDV, the HN protein was considered to play a major role in the tissue tropism (Huang et al., 2004). We systematically determined the tissue tropism of NDV by exchanging HN proteins between a velogenic (rSG10) or lentogenic (rLaSota) NDV strain and several other strains of different virulence. Our results showed that the viral tissue tropism was dependent on the origin of $\mathrm{HN}$ protein. The HN protein derived from the lentogenic virus within a backbone of a virulent strain exhibited a reduced tissue tropism and vice versa. These findings are in line with a previous study (Huang et al., 2004) and were slightly different from the results of several researchers (Oldoni et al., 2005; Wakamatsu et al., 2006). We think it is more reasonable that the $\mathrm{HN}$ protein derived from a virulent virus could increase and conversely that derived from a lentogenic virus can decrease the tissue tropism of the viruses. Generally, virulent strains are more infectious and can evidently enhance the biological activity of the chimeric virus (Dortmans et al., 2010; Paldurai et al., 2014; Jin et al., 2016).

The HN protein of NDV plays an important role in viral infection because it possesses the receptor recognition activity, while viral infection is initiated by attachment of the virion to the sialic acid-containing receptors on the surface of the target cell. HN protein can also promote fusion activity via its interaction with the F protein and act as an NA by removing sialic acid from progeny virus particles to prevent viral selfaggregation, aiding virus infection (Mirza et al., 1994; Huang et al., 2004; Melanson and Iorio, 2004; Heiden et al., 2014; Liu B. et al., 2015; Jin et al., 2016). Our results showed that HN proteins of the five selected NDV strains in this study had markedly different HAd, NA and fusogenic promotion activities at the protein level. Accordingly, the chimeric viruses also exhibited different biological activities, especially in receptor recognition and NA activities, which may be the major reason for the difference in viral replication among different chimeric viruses in vitro and in vivo. As we know, there is a positive correlation between viral pathogenicity and its replication efficiency (Bankamp et al., 2002; Dortmans et al., 2011; Jin et al., 2016). We think that the differing biological activity of $\mathrm{HN}$ proteins results in demonstration of different pathogenicities of the chimeric viruses in chickens. Furthermore, the HAd, NA and fusogenic promotion activities of $\mathrm{HN}$ proteins also influence the replication ability of NDV and thus the virulence.

Membrane fusion is necessary for NDV entry into host cells. The $\mathrm{F}$ protein is directly involved in membrane fusion and is a major determinant of NDV virulence, whereas the HN protein possesses fusion promotion activity by its interaction with the $\mathrm{F}$ protein and is therefore another determinant of viral virulence (Kim et al., 2011; Jin et al., 2016). Some recently published data showed that the stalk domains of the HN protein could promote the fusion event mediated by the F protein (Bose et al., 2012, 2014; Sun et al., 2015). The HN stalk domains included two relatively conserved heptad repeats (HR), with HR1 comprising residues 79-88 and HR2 comprising residues 96-110. The HR1 and HR2 regions are separated by an intervening region (IR) from residues 89-95 (Melanson and Iorio, 2004; Iorio et al., 2009; Yuan et al., 2015). Liu B. et al. (2015) found that A89Q and L94A mutations in the IR region weakened the fusion promotion activity of the HN protein. In this study, the rSG10 derivative rSG10-YuHN bearing the HN protein gene of rAPMV-2 Yucaipa exhibited significantly decreased fusogenic promotion activity $(p<0.001)$. Vero cells could not form syncytium after infection with rSG10-YuHN. Sequence analysis of the HN protein revealed that there was low amino acid sequence identity (33.4\%) between the APMV-2 Yucaipa and NDV SG10 strains, the residues at position 89 and 94 of IR region were also different between Yucaipa and other four viruses, which could be a possible reason for the decline of the fusion activity and virulence of the recombinant virus $\mathrm{rSG} 10-\mathrm{YuHN}$. It is also reported that the conformation of the HN stalk domain in other paramyxoviruses could influence the interaction of $\mathrm{HN}$ protein with $\mathrm{F}$ protein in the cell-cell fusion (Tsurudome et al., 2015). Whether this notion is applicable to NDV HN proteins needs to be further investigated.

\section{CONCLUSION}

Here we have comprehensively and systematically studied the correlation between the HN protein and NDV virulence. Using $\mathrm{HN}$ exchanges between a velogenic or lentogenic NDV strain and other strains of different virulence, we have demonstrated that alteration of the balance between receptor recognition, NA and fusion promotion activities causes changes in tissue tropism, replication and pathogenicity of the virus, which was closely related to the origin of the HN protein. Our study further highlights the importance of the $\mathrm{HN}$ glycoprotein in modulating the virulence of NDV. Further studies should concentrate on how to regulate the balance of the biological activities of the $\mathrm{HN}$ protein, which may 
provide a more complete understanding of the virulence of NDV and a more effective preventive approach for the disease.

\section{AUTHOR CONTRIBUTIONS}

Conceived and designed the experiments: J-hJ, J-lC, Z-rH, G-zZ. Performed the experiments: J-hJ, J-lC, Z-rH, Y-cR, $\mathrm{X}-\mathrm{hY}, \mathrm{YS}, \mathrm{H}-\mathrm{mY}, \mathrm{Y}-\mathrm{lY}$, and TL. Analyzed the data: J-hJ,

\section{REFERENCES}

Adu-Gyamfi, E., Kim, L. S., Jardetzky, T. S., and Lamb, R. A. (2016). Flexibility of the head-stalk linker domain of paramyxovirus $\mathrm{HN}$ glycoprotein is essential for triggering virus fusion. J. Virol. 90, 9172-9181. doi: 10.1128/JVI.01187-16

Alexander, D. J. (1982). Avian paramyxoviruses-other than Newcastle disease virus. Worlds Poult. Sci. J. 38, 97-104. doi: 10.1079/WPS19820008

Bankamp, B., Kearney, S. P., Liu, X., Bellini, W. J., and Rota, P. A. (2002). Activity of polymerase proteins of vaccine and wild-type measles virus strains in a minigenome replication assay. J. Virol. 76, 7073-7081. doi: 10.1128/JVI.76.14. 7073-7081.2002

Bose, S., Song, A. S., Jardetzky, T. S., and Lamb, R. A. (2014). Fusion activation through attachment protein stalk domains indicates a conserved core mechanism of paramyxovirus entry into cells. J. Virol. 88, 3925-3941. doi: 10.1128/JVI.03741-13

Bose, S., Zokarkar, A., Welch, B. D., Leser, G. P., Jardetzky, T. S., and Lamb, R. A. (2012). Fusion activation by a headless parainfluenza virus 5 hemagglutininneuraminidase stalk suggests a modular mechanism for triggering. Proc. Natl. Acad. Sci. U.S.A. 109, 2625-2634. doi: 10.1073/pnas.1213813109

Bratt, M. A., and Clavell, L. A. (1972). Hemolytic interaction of Newcastle disease virus and chicken erythrocytes. I. Quantitative comparison procedure. Appl. Microbiol. 23, 454-460.

Chhabra, R., Kuchipudi, S. V., Chantrey, J., and Ganapathy, K. (2016). Pathogenicity and tissue tropism of infectious bronchitis virus is associated with elevated apoptosis and innate immune responses. Virology 488, 232-241. doi: 10.1016/j.virol.2015.11.011

Connaris, H., Takimoto, T., Russell, R., Crennell, S., Moustafa, I., Portner, A., et al. (2002). Probing the sialic acid binding site of the hemagglutinin-neuraminidase of Newcastle disease virus: identification of key amino acids involved in cell binding, catalysis, and fusion. J. Virol. 76, 1816-1824. doi: 10.1128/JVI.76.4. 1816-1824.2002

Cornax, I., Diel, D. G., Rue, C. A., Estevez, C., Yu, Q., Miller, P. J., et al. (2013). Newcastle disease virus fusion and haemagglutinin-neuraminidase proteins contribute to its macrophage host range. J. Gen. Virol. 94, 1189-1194. doi: 10.1099/vir.0.048579-0

Crennell, S., Takimoto, T., Portner, A., and Taylor, G. (2000). Crystal structure of the multifunctional paramyxovirus hemagglutinin-neuraminidase. Nat. Struct. Biol. 7, 1068-1074. doi: 10.1038/81002

De Leeuw, O. S., Koch, G., Hartog, L., Ravenshorst, N., and Peeters, B. P. (2005). Virulence of Newcastle disease virus is determined by the cleavage site of the fusion protein and by both the stem region and globular head of the haemagglutinin-neuraminidase protein. J. Gen. Virol. 86, 1759-1769. doi: 10.1099/vir.0.80822-0

Dimitrov, K. M., Ramey, A. M., Qiu, X., Bahl, J., and Afonso, C. L. (2016). Temporal, geographic, and host distribution of avian paramyxovirus 1 (Newcastle disease virus). Infect. Genet. Evol. 39, 22-34. doi: 10.1016/j.meegid. 2016.01.008

Dortmans, J. C., Koch, G., Rottier, P. J., and Peeters, B. P. (2009). Virulence of pigeon paramyxovirus type 1 does not always correlate with the cleavability of its fusion protein. J. Gen. Virol. 90, 2746-2750. doi: 10.1099/vir.0.014118-0

Dortmans, J. C., Koch, G., Rottier, P. J., and Peeters, B. P. (2011). Virulence of Newcastle disease virus: What is known so far? Vet. Res. 42:122. doi: 10.1186/ 1297-9716-42-122

Dortmans, J. C., Rottier, P. J., Koch, G., and Peeters, B. P. (2010). The viral replication complex is associated with the virulence of Newcastle disease virus. J. Virol. 84, 10113-10120. doi: 10.1128/JVI.00097-10
J-lC, Z-rH, X-hY, and G-zZ. Contributed reagents/materials/ analysis tools: G-ZZ. Wrote the paper: J-hJ, J-lC, Z-rH, and G-zZ.

\section{FUNDING}

This study was supported by the Beijing Agriculture Innovation Consortium of Poultry Research System (BAIC04-2017).

Glickman, R. L., Syddall, R. J., Iorio, R. M., Sheehan, J. P., and Bratt, M. A. (1988). Quantitative basic residue requirements in the cleavage-activation site of the fusion glycoprotein as a determinant of virulence for Newcastle disease virus. J. Virol. 62, 354-356.

Haller, B. L., Barkon, M. L., Vogler, G. P., and Virgin, H. W. (1995). Genetic mapping of reovirus virulence and organ tropism in severe combined immunodeficient mice: organ-specific virulence genes. J. Virol. 69, 357-364.

Heiden, S., Grund, C., Röder, A., Granzow, H., Kühnel, D., Mettenleiter, T. C., et al. (2014). Different regions of the Newcastle disease virus fusion protein modulate pathogenicity. PLoS ONE 9:e113344. doi: 10.1371/journal.pone.0113344

Huang, Z., Krishnamurthy, S., Panda, A., and Samal, S. K. (2003). Newcastle disease virus $\mathrm{V}$ protein is associated with viral pathogenesis and functions as an alpha interferon antagonist. J. Virol. 77, 8676-8685. doi: 10.1128/JVI.77.16. 8676-8685.2003

Huang, Z., Panda, A., Elankumaran, S., Govindarajan, D., Rockemann, D. D., and Samal, S. K. (2004). The hemagglutinin-neuraminidase protein of Newcastle disease virus determines tropism and virulence. J. Virol. 78, 4176-4184. doi: 10.1128/JVI.78.8.4176-4184.2004

Iorio, R. M., Field, G. M., Sauvron, J. M., Mirza, A. M., Deng, R., Mahon, P. J., et al. (2001). Structural and functional relationship between the receptor recognition and neuraminidase activities of the Newcastle disease virus hemagglutininneuraminidase protein: receptor recognition is dependent on neuraminidase activity. J. Virol. 75, 1918-1927. doi: 10.1128/JVI.75.4.1918-1927.2001

Iorio, R. M., Melanson, V. R., and Mahon, P. J. (2009). Glycoprotein interactions in paramyxovirus fusion. Future Virol. 4, 335-351. doi: 10.2217/fvl.09.17

Jin, J., Zhao, J., Ren, Y., Zhong, Q., and Zhang, G. (2016). Contribution of HN protein length diversity to Newcastle disease virus virulence, replication and biological activities. Sci. Rep. 6:36890. doi: 10.1038/srep36890

Khattar, S. K., Yan, Y., Panda, A., Collins, P. L., and Samal, S. K. (2009). A Y526Q mutation in the Newcastle disease virus HN protein reduces its functional activities and attenuates virus replication and pathogenicity. J. Virol. 83, 7779-7782. doi: 10.1128/JVI.00536-09

Kim, S. H., Subbiah, M., Samuel, A. S., Collins, P. L., and Samal, S. K. (2011). Roles of the fusion and hemagglutinin-neuraminidase proteins in replication, tropism, and pathogenicity of avian paramyxoviruses. J. Virol. 85, 8582-8596. doi: 10.1128/JVI.00652-11

Li, J., Quinlan, E., Mirza, A., and Iorio, R. M. (2004). Mutated form of the Newcastle disease virus hemagglutinin-neuraminidase interacts with the homologous fusion protein despite deficiencies in both receptor recognition and fusion promotion. J. Virol. 78, 5299-5310. doi: 10.1128/JVI.78.10.5299-5310.2004

Liu, B., Ji, Y., Lin, Z., Fu, Y., Muhammad Dafallah, R., and Zhu, Q. (2015). Two single amino acid substitutions in the intervening region of Newcastle disease virus HN protein attenuate viral replication and pathogenicity. Sci. Rep. 5:13038. doi: 10.1038/srep13038

Liu, M. M., Cheng, J. L., Yu, X. H., Qin, Z. M., Tian, F. L., and Zhang, G. Z. (2015). Generation by reverse genetics of an effective attenuated Newcastle disease virus vaccine based on a prevalent highly virulent Chinese strain. Biotechnol. Lett. 37, 1287-1296. doi: 10.1007/s10529-015-1799-Z

Madansky, C. H., and Bratt, M. A. (1978). Noncytopathic mutants of Newcastle disease virus. J. Virol. 26, 724-729.

Mayo, M. (2002). A summary of taxonomic changes recently approved by ICTV. Arch. Virol. 147, 1655-1656. doi: 10.1007/s007050200039

Melanson, V. R., and Iorio, R. M. (2004). Amino acid substitutions in the F-specific domain in the stalk of the Newcastle disease virus HN protein modulate fusion and interfere with its interaction with the F protein. J. Virol. 78, 13053-13061. doi: 10.1128/JVI.78.23.13053-13061.2004 
Meliopoulos, V. A., Karlsson, E. A., Kercher, L., Cline, T., Freiden, P., Duan, S., et al. (2014). Human H7N9 and H5N1 influenza viruses differ in induction of cytokines and tissue tropism. J. Virol. 88, 12982-12991. doi: 10.1128/JVI. 01571-14

Miller, P. J., Kim, L. M., Ip, H. S., and Afonso, C. L. (2009). Evolutionary dynamics of Newcastle disease virus. Virology 391, 64-72. doi: 10.1016/j.virol.2009.05.033

Miller, P. J., and Koch, G. (2013). "Newcastle disease, other avian paramyxoviruses and avian metapneumovirus infections," in Diseases of Poultry, 13th Edn, eds D. E. Swayne, J. R. Glisson, L. R. McDougald, L. K. Nolan, D. L. Suarez, and N. Venugopal (Ames, IA: Wiley-Blackwell), 89-138.

Mirza, A. M., Deng, R., and Iorio, R. M. (1994). Site-directed mutagenesis of a conserved hexapeptide in the paramyxovirus hemagglutinin-neuraminidase glycoprotein: effects on antigenic structure and function. J. Virol. 68, 5093-5099.

Morrison, T. G. (2001). The three faces of paramyxovirus attachment proteins. Trends Microbiol. 9, 103-105. doi: 10.1016/S0966-842X(01)01959-X

Munir, M., Cortey, M., Abbas, M., Qureshi, Z. U., Afzal, F., Shabbir, M. Z., et al. (2012). Biological characterization and phylogenetic analysis of a novel genetic group of Newcastle disease virus isolated from outbreaks in commercial poultry and from backyard poultry flocks in Pakistan. Infect. Genet. Evol. 12, 1010-1019. doi: 10.1016/j.meegid.2012.02.015

Nagai, Y., Klenk, H. D., and Rott, R. (1976). Proteolytic cleavage of the viral glycoproteins and its significance for the virulence of Newcastle disease virus. Virology 72, 494-508. doi: 10.1016/0042-6822(76)90178-1

Oldoni, I., Brown, C. C., King, D. J., Samal, S., and Seal, B. S. (2005). The use of in situ hybridization and immunohistochemistry to study the pathogenesis of various Newcastle disease virus strains and recombinants in embryonated chicken eggs. Microb. Pathog. 39, 69-75. doi: 10.1016/j.micpath.2005.04.002

Paldurai, A., Kim, S. H., Nayak, B., Xiao, S., Shive, H., Collins, P. L., et al. (2014). Evaluation of the contributions of the individual viral genes to Newcastle disease virus virulence and pathogenesis. J. Virol. 88, 8579-8596. doi: 10.1128/ JVI.00666- 14

Panda, A., Huang, Z., Elankumaran, S., Rockemann, D. D., and Samal, S. K. (2004). Role of fusion protein cleavage site in the virulence of Newcastle disease virus. Microb. Pathog. 36, 1-10. doi: 10.1016/j.micpath.2003.07.003

Peeters, B. P. H., de Leeuw, O. S., Koch, G., and Gielkens, A. L. J. (1999). Rescue of Newcastle disease virus from cloned cDNA: evidence that cleavability of the fusion protein is a major determinant for virulence. J. Virol. 73, 5001-5009.

Porotto, M., Salah, Z., DeVito, I., Talekar, A., Palmer, S. G., Xu, R., et al. (2012). The second receptor binding site of the globular head of the Newcastle disease virus hemagglutinin- neuraminidase activates the stalk of multiple paramyxovirus receptor binding proteins to trigger fusion. J. Virol. 86, 5730-5741. doi: 10.1128/ JVI.06793-11

Potier, M., Mameli, L., Belisle, M., Dallaire, L., and Melancon, S. (1979). Fluorometric assay of neuraminidase with a sodium (4-methylumbelliferyl- $\alpha$ D-N-acetylneuraminate) substrate. Anal. Biochem. 94, 287-296. doi: 10.1016/ 0003-2697(79)90362-2

Reed, L. J., and Muench, H. (1938). A simple method of estimating fifty per cent endpoints. Am. J. Epidemiol. 27, 493-497. doi: 10.1093/oxfordjournals.aje. a1 18408
Rout, S. N., and Samal, S. K. (2008). The large polymerase protein is associated with the virulence of Newcastle disease virus. J. Virol. 82, 7828-7836. doi: 10.1128/JVI.00578-08

Sun, C., Wen, H., Chen, Y., Chu, F., Lin, B., Ren, G., et al. (2015). Roles of the highly conserved amino acids in the globular head and stalk region of the Newcastle disease virus HN protein in the membrane fusion process. Biosci. Trends 9, 56-64. doi: 10.5582/bst.2014.01140

Toyoda, T., Sakaguchi, T., Imai, K., Inocencio, N. M., Gotoh, B., Hamaguchi, M., et al. (1987). Structural comparison of the cleavage-activation site of the fusion glycoprotein between virulent and avirulent strains of Newcastle disease virus. Virology 158, 242-247. doi: 10.1016/0042-6822(87) 90261-3

Tsurudome, M., Ito, M., Ohtsuka, J., Hara, K., Komada, H., Nishio, M., et al. (2015). The fusion protein specificity of the parainfluenza virus hemagglutinin-neuraminidase protein is not solely defined by the primary structure of its stalk domain. J. Virol. 89, 12374-12387. doi: 10.1128/JVI. 01448-15

Wakamatsu, N., King, D. J., Seal, B. S., Samal, S. K., and Brown, C. C. (2006). The pathogenesis of Newcastle disease: a comparison of selected Newcastle disease virus wild-type strains and their infectious clones. Virology 353, 333-343. doi: 10.1016/j.virol.2006.06.013

Yu, X. H., Cheng, J. L., Xue, J., Jin, J. H., Song, Y., Zhao, J., et al. (2017). Roles of the polymerase-associated protein genes in Newcastle disease virus virulence. Front. Microbiol. 8:161. doi: 10.3389/fmicb.2017. 00161

Yuan, P., Paterson, R. G., Leser, G. P., Lamb, R. A., and Jardetzky, T. S. (2012). Structure of the Ulster strain Newcastle disease virus hemagglutininneuraminidase reveals auto-inhibitory interactions associated with low virulence. PLoS Pathog. 8:e1002855. doi: 10.1371/journal.ppat.1002855

Yuan, P., Swanson, K. A., Leser, G. P., Paterson, R. G., Lamb, R. A., and Jardetzky, T. S. (2015). Structure of the Newcastle disease virus hemagglutininneuraminidase (HN) ectodomain reveals a four-helix bundle stalk. Proc. Natl. Acad. Sci. U.S.A. 108, 14920-14925. doi: 10.1073/pnas.1111691108

Zaitsev, V., von Itzstein, M., Groves, D., Kiefel, M., Takimoto, T., Portner, A., et al. (2004). Second sialic acid binding site in Newcastle disease virus hemagglutininneuraminidase: implications for fusion. J. Virol. 78, 3733-3741. doi: 10.1128/ JVI.78.7.3733-3741.2004

Conflict of Interest Statement: The authors declare that the research was conducted in the absence of any commercial or financial relationships that could be construed as a potential conflict of interest.

Copyright (c) 2017 Jin, Cheng, He, Ren, Yu, Song, Yang, Yang, Liu and Zhang. This is an open-access article distributed under the terms of the Creative Commons Attribution License (CC BY). The use, distribution or reproduction in other forums is permitted, provided the original author(s) or licensor are credited and that the original publication in this journal is cited, in accordance with accepted academic practice. No use, distribution or reproduction is permitted which does not comply with these terms. 